TRANSACTIONS OF THE

AMERICAN MATHEMATICAL SOCIETY

Volume 360, Number 12, December 2008, Pages 6555-6572

S 0002-9947(08)04515-7

Article electronically published on July 22, 2008

\title{
WELL-POSEDNESS FOR THE KADOMTSEV-PETVIASHVILI II EQUATION AND GENERALISATIONS
}

\author{
MARTIN HADAC
}

\begin{abstract}
We show the local in time well-posedness of the Cauchy problem for the Kadomtsev-Petviashvili II equation for initial data in the non-isotropic Sobolev space $H^{s_{1}, s_{2}}\left(\mathbb{R}^{2}\right)$ with $s_{1}>-\frac{1}{2}$ and $s_{2} \geq 0$. On the $H^{s_{1}, 0}\left(\mathbb{R}^{2}\right)$ scale this result includes the full subcritical range without any additional low frequency assumption on the initial data. More generally, we prove the local in time well-posedness of the Cauchy problem for the following generalisation of the KP II equation:

$$
\left(u_{t}-\left|D_{x}\right|^{\alpha} u_{x}+\left(u^{2}\right)_{x}\right)_{x}+u_{y y}=0, \quad u(0)=u_{0},
$$

for $\frac{4}{3}<\alpha \leq 6, s_{1}>\max \left(1-\frac{3}{4} \alpha, \frac{1}{4}-\frac{3}{8} \alpha\right), s_{2} \geq 0$ and $u_{0} \in H^{s_{1}, s_{2}}\left(\mathbb{R}^{2}\right)$. We deduce global well-posedness for $s_{1} \geq 0, s_{2}=0$ and real valued initial data.
\end{abstract}

\section{INTRODUCTION}

In this paper we study the Cauchy problem for the Kadomtsev-Petviashvili II equation

$$
\left(u_{t}+u_{x x x}+\left(u^{2}\right)_{x}\right)_{x}+u_{y y}=0 \quad \text { in } \mathbb{R}^{3}, \quad u(0)=u_{0} .
$$

The Kadomtsev-Petviashvili II equation, as well as the Kadomtsev-Petviashvili I equation $\left(u_{t}+u_{x x x}+\left(u^{2}\right)_{x}\right)_{x}-u_{y y}=0$, are two-dimensional extensions of the Korteweg-de-Vries equation; see [8]. More generally, we will consider the following dispersion generalised Kadomtsev-Petviashvili II type equation:

$$
\left(u_{t}-\left|D_{x}\right|^{\alpha} u_{x}+\left(u^{2}\right)_{x}\right)_{x}+u_{y y}=0 \quad \text { in } \mathbb{R}^{3}, \quad u(0)=u_{0}
$$

where $\frac{4}{3}<\alpha \leq 6$. Here $\left|D_{x}\right|^{\alpha}$ is the Fourier multiplier operator with multiplier $|\xi|^{\alpha}$. We consider initial values $u_{0}$ in the non-isotropic Sobolev spaces

$$
H^{s_{1}, s_{2}}\left(\mathbb{R}^{2}\right):=\left\{u_{0} \in \mathcal{S}^{\prime}\left(\mathbb{R}^{2}\right) \mid\left\|u_{0}\right\|_{H^{s_{1}, s_{2}}}:=\left\|\langle\xi\rangle^{s_{1}}\langle\eta\rangle^{s_{2}} \widehat{u_{0}}\right\|_{L_{\xi \eta}^{2}}<\infty\right\} .
$$

Note that the case $\alpha=2$ of (1.2) is (1.1), whereas the case $\alpha=4$ is known as the fifth order KP II equation

$$
\left(u_{t}-u_{x x x x x}+\left(u^{2}\right)_{x}\right)_{x}+u_{y y}=0 \quad \text { in } \mathbb{R}^{3}, \quad u(0)=u_{0} .
$$

Received by the editors January 22, 2007.

2000 Mathematics Subject Classification. Primary 35Q53; Secondary 35B30.

Key words and phrases. Kadomtsev-Petviashvili II equation, Cauchy problem, local wellposedness.

The research for this work was mainly carried out while the author was employed at the Department of Mathematics of the University of Dortmund.

(C)2008 American Mathematical Society
Reverts to public domain 28 years from publication 
We are interested in low regularity well-posedness of (1.2). By using refined Fourier restriction norm spaces we will prove new bilinear estimates which allow us to apply the contraction mapping principle.

In the seminal work [3] Bourgain shows the (global) well-posedness of (1.1) (on $\mathbb{T}^{2}$ rather than on $\mathbb{R}^{2}$ ) with initial values in $L^{2}$, i.e. for $s_{1}=s_{2}=0$. This result has been improved afterwards by Takaoka and Tzvetkov [17] and Isaza and Mejía 6] to the local in time well-posedness of (1.1) for $s_{1}>-\frac{1}{3}$ and $s_{2} \geq 0$. (For previous results see also [18, [19, 15].) In [16] Takaoka shows local wellposedness for $s_{1}>-\frac{1}{2}, s_{2}=0$, but only if the additional low frequency condition $\left|D_{x}\right|^{-\frac{1}{2}+\varepsilon} u_{0} \in L^{2}$ (with suitably chosen $\varepsilon$ ) is imposed on the initial value.

For the fifth order KP-II equation (1.4) local well-posedness was shown by Saut and Tzvetkov (see [13, 14]) for $s_{1} \geq-\frac{1}{4}$ and $s_{2} \geq 0$. (Note that the equation considered in [13, 14] is slightly more general than (1.4) because it also contains the third order term.) Very recently, Isaza, López and Mejía [7] improved the local well-posedness result to $s_{1}>-\frac{5}{4}$ and $s_{2} \geq 0$. (These authors also showed global well-posedness of (1.4) for $s_{1}>-\frac{4}{7}$ and $s_{2}=0$.)

For general $\alpha \in\left(\frac{4}{3}, 6\right]$ Iório and Nunes [5] showed the local well-posedness for initial values $u_{0}$ in the isotropic Sobolev space $H^{s}\left(\mathbb{R}^{2}\right), s>2$, with the additional low frequency condition $\partial_{x}^{-1} u_{0} \in H^{s}\left(\mathbb{R}^{2}\right)$ using parabolic regularization. Let us note that they consider much more general equations and do not use the dispersive structure of the equation.

For a recent result concerning the so-called mass constraint property for solutions of equations of type (1.2), see [11].

Our main result for equation (1.1) is the following.

Theorem 1.1. Let $s_{1}>-\frac{1}{2}$ and $s_{2} \geq 0$. For $R>0$ there exists $T=T(R)>0$ and a Banach space $X_{T} \hookrightarrow C\left([-T, T] ; H^{s_{1}, s_{2}}\left(\mathbb{R}^{2}\right)\right)$, such that for every $u_{0} \in B_{R}:=$ $\left\{u_{0} \in H^{s_{1}, s_{2}}\left(\mathbb{R}^{2}\right) \mid\left\|u_{0}\right\|_{H^{s_{1}, s_{2}}\left(\mathbb{R}^{2}\right)}<R\right\}$ there is exactly one solution $u$ of equation (1.1) in $X_{T}$. Furthermore the mapping $F_{R}: B_{R} \rightarrow X_{T}, u_{0} \mapsto u$ is analytic.

More generally, we will show the following theorem concerning equation (1.2).

Theorem 1.2. Let $\frac{4}{3}<\alpha \leq 6, s_{1}>\max \left(1-\frac{3}{4} \alpha, \frac{1}{4}-\frac{3}{8} \alpha\right)$ and $s_{2} \geq 0$. For $R>0$ there exists $T=T(R)>0$ and a Banach space $X_{T} \hookrightarrow C\left([-T, T] ; H^{s_{1}, s_{2}}\left(\mathbb{R}^{2}\right)\right)$, such that for every $u_{0} \in B_{R}:=\left\{u_{0} \in H^{s_{1}, s_{2}}\left(\mathbb{R}^{2}\right) \mid\left\|u_{0}\right\|_{H^{s_{1}, s_{2}}\left(\mathbb{R}^{2}\right)}<R\right\}$ there is exactly one solution $u$ of equation (1.2) in $X_{T}$. Furthermore the mapping $F_{R}: B_{R} \rightarrow$ $X_{T}, u_{0} \mapsto u$ is analytic.

Remark 1.3. If we (formally) apply the operator $\partial_{x}^{-1}$ to equation (1.2) and use Duhamel's formula, equation (1.2) is (for suitable $u$ ) equivalent to the integral equation

$$
u(t)=U_{\alpha}(t) u_{0}-\int_{0}^{t} U_{\alpha}\left(t-t^{\prime}\right) \partial_{x}\left(u\left(t^{\prime}\right)^{2}\right) d t^{\prime}
$$

where $U_{\alpha}$ is the unitary group on $H^{s_{1}, s_{2}}\left(\mathbb{R}^{2}\right)$ defined by

$$
\widehat{U_{\alpha}(t) u_{0}}(\xi, \eta):=\exp \left(i t\left(\xi|\xi|^{\alpha}-\frac{\eta^{2}}{\xi}\right)\right) \widehat{u_{0}}(\xi, \eta)
$$

We define a solution of (1.2) in $X_{T}$ (for $T \leq 1$ ) to be a solution of the operator equation

$$
u(t)=\psi(t) U_{\alpha}(t) u_{0}-\Gamma_{T}(u, u)(t), \quad t \in[-T, T]
$$


where $\Gamma_{T}$ is the bilinear operator on $X_{T}$ defined for smooth $u_{1}, u_{2}$ by

$$
\Gamma_{T}\left(u_{1}, u_{2}\right)(t):=\psi_{T}(t) \int_{0}^{t} U_{\alpha}\left(t-t^{\prime}\right) \partial_{x}\left(u_{1} u_{2}\right)\left(t^{\prime}\right) d t^{\prime}
$$

and $\psi \in C_{0}^{\infty}(\mathbb{R})$ is a cut-off function with $\psi(t)=1$ for $|t| \leq 1$ and $\psi(t)=0$ for $|t| \geq 2$. Furthermore $\psi_{T}(t)=\psi(t / T)$.

Remark 1.4. In the particular case $\alpha=4$ of the fifth order KP II equation, Theorem 1.2 shows the local well-posedness of (1.4) for $s_{1}>-\frac{5}{4}$ and $s_{2} \geq 0$. We therefore get a local well-posedness result for the same class of initial data as Isaza, López and Mejía in [7]. Note, though, that the spaces $X_{T}$, where the local wellposedness result of Theorem 1.2 holds true, are different from those used in [7] (see Remark 4.4).

Remark 1.5. Let us note that if $u$ is a solution of (1.2), then so is

$$
u_{\lambda}(t, x, y)=\lambda^{\alpha} u\left(\lambda^{\alpha+1} t, \lambda x, \lambda^{\frac{\alpha}{2}+1} y\right) .
$$

Considering the homogeneous Sobolev norm

$$
\left\|u_{0}\right\|_{\dot{H}^{s_{1}, s_{2}}}:=\left\||\xi|^{s_{1}}|\eta|^{s_{2}} \widehat{u_{0}}\right\|_{L_{\xi, \eta}^{2}}
$$

we get $\left\|u_{\lambda}(0, \cdot, \cdot)\right\|_{\dot{H}^{s_{1}, s_{2}}}=\lambda^{\frac{3}{4} \alpha-1+s_{1}+\left(1+\frac{\alpha}{2}\right) s_{2}}\|u(0, \cdot, \cdot)\|_{\dot{H}^{s_{1}, s_{2}}}$. This argument suggests that we get ill-posedness for $s_{1}+\left(1+\frac{\alpha}{2}\right) s_{2}<1-\frac{3}{4} \alpha$. Note that for $\frac{4}{3}<\alpha \leq 2$ and $s_{2}=0$ we reach the critical value $1-\frac{3}{4} \alpha$ of $s_{1}$, except for the endpoint. For $\alpha=2$ it is proved in 10, Theorem 4.2, that the flow cannot be $C^{3}$ at the origin from $H^{s_{1}, 0}\left(\mathbb{R}^{2}\right)$ to $H^{s_{1}, 0}\left(\mathbb{R}^{2}\right)$ for $s_{1}<-\frac{1}{2}$, so that our result is sharp (except at the endpoint) for the scale $H^{s_{1}, 0}\left(\mathbb{R}^{2}\right)$ in the sense of $C^{3}$-well-posedness. (Note that while in general the space $H^{s_{1}, s_{2}}\left(\mathbb{R}^{2}\right)$ defined in 10 differs from the one defined in (1.3), they coincide for $s_{2}=0$.) For $\alpha>2$, though, we have that $\frac{1}{4}-\frac{3}{8} \alpha>1-\frac{3}{4} \alpha$, so that we do not reach the scaling limit in this case.

By combining the local well-posedness result of Theorem 1.2 with the conservation of the $L^{2}$-norm which holds for real valued solutions of (1.7), we get the following global result, where $H^{s_{1}, 0}\left(\mathbb{R}^{2} ; \mathbb{R}\right)$ denotes the subspace of all real valued functions in $H^{s_{1}, 0}\left(\mathbb{R}^{2}\right)$.

Theorem 1.6. Let $\frac{4}{3}<\alpha \leq 6, s_{1} \geq 0$ and $T>0$. Then there exists a Banach space $X_{T} \hookrightarrow C\left([-T, T] ; H^{s_{1}, 0}\left(\mathbb{R}^{2} ; \mathbb{R}\right)\right)$, such that for every $u_{0} \in H^{s_{1}, 0}\left(\mathbb{R}^{2} ; \mathbb{R}\right)$ there is exactly one solution $u$ of equation (1.2) in $X_{T}$.

Let us fix some notation we use throughout the paper:

- For $\xi \in \mathbb{R}$ let $\langle\xi\rangle:=\left(1+|\xi|^{2}\right)^{\frac{1}{2}}$.

- For $u \in \mathcal{S}^{\prime}\left(\mathbb{R}^{n}\right)$ the Fourier transformation of $u$ in $\mathbb{R}^{n}$ is denoted by $\widehat{u}$ or $\mathcal{F} u$. A partial Fourier transformation, with respect to some of the $n$ variables, is denoted for example by $\mathcal{F}_{1}$ for the Fourier transformation in the first variable, etc.

- $\mu=(\tau, \xi, \eta) \in \mathbb{R}^{3}$ always denotes the Fourier variable dual to $(t, x, y)$.

- For $\mu=(\tau, \xi, \eta)$ let $\lambda:=\lambda(\mu):=\tau-\xi|\xi|^{\alpha}+\frac{\eta^{2}}{\xi}$. If there are two frequency variables $\mu$ and $\mu_{1}$ we write $\lambda_{1}:=\lambda\left(\mu_{1}\right), \lambda_{2}:=\lambda\left(\mu-\mu_{1}\right)$ and $\left|\lambda_{\max }\right|=$ $\max \left(|\lambda|,\left|\lambda_{1}\right|,\left|\lambda_{2}\right|\right)$ for short. Also let $\left|\xi_{\max }\right|:=\max \left(|\xi|,\left|\xi_{1}\right|,\left|\xi-\xi_{1}\right|\right)$. Let $\left|\xi_{\text {min }}\right|$ and $\left|\xi_{\text {med }}\right|$ be defined analogously.

- $A \lesssim B$ means that there is a (harmless) constant $C$ such that $A \leq C B$. 
- For $X$ and $Y$ the Banach spaces $X \hookrightarrow Y$ mean that there is a continuous embedding from $X$ into $Y$. Furthermore $C_{b}(\mathbb{R} ; X)$ denotes the space of all continuous and bounded functions $f: \mathbb{R} \rightarrow X$ with the sup-norm.

The author would like to thank S. Herr and H. Koch for valuable discussions and suggestions on the subject. He would also like to thank the anonymous referee for useful remarks concerning the presentation of the proof.

\section{Definition OF THE SOlution SPACES}

Definition 2.1. Let us consider the following space of test functions:

$$
\tilde{\mathcal{S}}:=\left\{\phi \in \mathcal{S}\left(\mathbb{R}^{3}\right) \mid \partial_{\xi}^{k} \widehat{\phi}(\tau, 0, \eta)=0 \forall k \in \mathbb{N}_{0} \forall(\tau, \eta) \in \mathbb{R}^{2}\right\} .
$$

For $s_{1}, s_{2}, b \in \mathbb{R}, \sigma \geq 0$ and $\phi \in \tilde{\mathcal{S}}$ define

$$
\|\phi\|_{X_{\sigma}^{s_{1}, s_{2}, b}}:=\left\||\xi|^{-\sigma}\langle\xi\rangle^{s_{1}+\sigma}\langle\eta\rangle^{s_{2}}\langle\lambda\rangle^{b} \widehat{\phi}\right\|_{L_{\mu}^{2}} .
$$

Let $X_{\sigma}^{s_{1}, s_{2}, b}$ be the completion of $\tilde{\mathcal{S}}$ with respect to the norm (2.2).

Functions in $\tilde{\mathcal{S}}$ have the property that for every $k \in \mathbb{N}_{0}$ there is a $C_{k}>0$ such that $|\widehat{\phi}(\tau, \xi, \eta)| \leq C_{k}|\xi|^{k}$ for all $(\tau, \xi, \eta) \in \mathbb{R}^{3}$. This property ensures that the right hand side of (2.2) is well defined in spite of the singularity along $\xi=0$ in the term $\lambda=\tau-\xi|\xi|^{\alpha}+\frac{\eta^{2}}{\xi}$ and the factor $|\xi|^{-\sigma}$.

Remark 2.2. At least for $b>-\frac{1}{2}-\sigma$, we can identify $X_{\sigma}^{s_{1}, s_{2}, b}$ with the subspace of tempered distributions $u$ on $\mathbb{R}^{3}$ such that $\widehat{u}$ is a regular distribution and $|\xi|^{-\sigma}\langle\xi\rangle^{s_{1}+\sigma}\langle\eta\rangle^{s_{2}}\langle\lambda\rangle^{b} \widehat{u} \in L^{2}$.

Remark 2.3. If $s_{2}=0$ we write $X_{\sigma}^{s_{1}, b}$ for short instead of $X_{\sigma}^{s_{1}, s_{2}, b}$.

Remark 2.4. The spaces $X_{\sigma}^{s_{1}, s_{2}, b}$ are modifications of spaces first used by Bourgain [1, 2] in the context of the KdV and Schrödinger equations.

We have the following well-known linear estimates.

Proposition 2.5. For $b \geq 0$ and $s_{1}, s_{2} \in \mathbb{R}$ we have

$$
\left\|\psi U_{\alpha}(t) u_{0}\right\|_{X_{0}^{s_{1}, s_{2}, b}} \lesssim\left\|u_{0}\right\|_{H^{s_{1}, s_{2}\left(\mathbb{R}^{2}\right)}} .
$$

Proof. See for example [4].

Proposition 2.6. For $-\frac{1}{2}<b^{\prime} \leq 0 \leq b \leq b^{\prime}+1, T \leq 1$ and $s_{1}, s_{2} \in \mathbb{R}$ we have

$$
\left\|\psi_{T} \int_{0}^{t} U_{\alpha}\left(t-t^{\prime}\right) F\left(t^{\prime}\right) d t^{\prime}\right\|_{X_{\sigma}^{s_{1}, s_{2}, b}} \lesssim T^{1-\left(b-b^{\prime}\right)}\|F\|_{X_{\sigma}^{s_{1}, s_{2}, b^{\prime}}} .
$$

Proof. For $\sigma=0$ see 4 . For $\sigma \neq 0$ consider the operator $I_{\sigma}$ defined for $u \in \tilde{\mathcal{S}}$ by $\left(\mathcal{F}_{2} I_{\sigma} u\right)(t, \xi, y)=\left(\frac{\langle\xi\rangle}{|\xi|}\right)^{\sigma} \mathcal{F}_{2} u(t, \xi, y)$. Then $I_{\sigma}: X_{\sigma}^{s_{1}, s_{2}, b} \rightarrow X_{0}^{s_{1}, s_{2}, b}$ is an isometric isomorphism. Therefore we have

$$
\begin{aligned}
\left\|\psi_{T} \int_{0}^{t} U_{\alpha}\left(t-t^{\prime}\right) F\left(t^{\prime}\right) d t^{\prime}\right\|_{X_{\sigma}^{s_{1}, s_{2}, b^{\prime}}} & =\left\|I_{\sigma} \psi_{T} \int_{0}^{t} U_{\alpha}\left(t-t^{\prime}\right) F\left(t^{\prime}\right) d t^{\prime}\right\|_{X_{0}^{s_{1}, s_{2}, b^{\prime}}} \\
& =\left\|\psi_{T} \int_{0}^{t} U_{\alpha}\left(t-t^{\prime}\right) I_{\sigma} F\left(t^{\prime}\right) d t^{\prime}\right\|_{X_{0}^{s_{1}, s_{2}, b^{\prime}}} \\
& \lesssim T^{1-\left(b-b^{\prime}\right)}\left\|I_{\sigma} F\right\|_{X_{0}^{s_{1}, s_{2}, b}} \\
& =T^{1-\left(b-b^{\prime}\right)}\|F\|_{X_{\sigma}^{s_{1}, s_{2}, b}}
\end{aligned}
$$


We have the following well-known embedding result for the $X_{\sigma}^{s_{1}, s_{2}, b}$-spaces.

Proposition 2.7. Let $s_{1}, s_{2} \in \mathbb{R}, \sigma \geq 0$ and $b>\frac{1}{2}$. Then

$$
X_{\sigma}^{s_{1}, s_{2}, b} \hookrightarrow C_{b}\left(\mathbb{R} ; H^{s_{1}, s_{2}}\left(\mathbb{R}^{2}\right)\right) .
$$

Proof. For $\sigma=0$ see [4]. But for $\sigma>0$ we have that $\|u\|_{X_{0}^{s_{1}, s_{2}, b}} \leq\|u\|_{X_{\sigma}^{s_{1}, s_{2}, b}}$.

Definition 2.8. Let $X \hookrightarrow C_{b}\left(\mathbb{R} ; H^{s_{1}, s_{2}}\left(\mathbb{R}^{2}\right)\right)$. Then we define the restriction norm space $X_{T}:=\left\{\left.u\right|_{[-T, T]} \mid u \in X\right\}$ with the norm

$$
\|u\|_{X_{T}}=\inf \left\{\|\tilde{u}\|_{X}|\tilde{u}|_{[-T, T]}=u\right\} .
$$

Then $X_{T} \hookrightarrow C\left([-T, T] ; H^{s_{1}, s_{2}}\left(\mathbb{R}^{2}\right)\right)$.

\section{Strichartz and Refined Strichartz estimates}

Exactly as in the case $\alpha=2$ (see [12]) we show the following Strichartz estimates for the solution of the linear equation. For the convenience of the reader we will give the full proof here.

Theorem 3.1. Let $2<q \leq \infty, \frac{1}{r}+\frac{1}{q}=\frac{1}{2}$ and $\gamma:=\left(1-\frac{2}{r}\right)\left(\frac{1}{2}-\frac{\alpha}{4}\right)$. Then we have

$$
\left\|\left|D_{x}\right|^{-\gamma} U_{\alpha}(t) u_{0}\right\|_{L_{t}^{q} L_{x y}^{r}} \lesssim\left\|u_{0}\right\|_{L_{x y}^{2}} .
$$

Proof. Let $\theta:=1-\frac{\alpha}{2}$ and $\kappa \in \mathbb{R}$. As $\theta<1$, we have that

$$
m_{t}(\xi, \eta):=|\xi|^{-\theta+i \kappa} e^{i t\left(\xi|\xi|^{\alpha}-\frac{\eta^{2}}{\xi}\right)} \in \mathcal{S}^{\prime}\left(\mathbb{R}^{2}\right)
$$

for every $t \in \mathbb{R}$. Therefore we have for $u_{0} \in \mathcal{S}\left(\mathbb{R}^{2}\right)$ and $t \in \mathbb{R}$

$$
\left|D_{x}\right|^{-\theta+i \kappa} U_{\alpha}(t) u_{0}=\mathcal{F}^{-1}\left(m_{t} \widehat{u_{0}}\right)=\mathcal{F}^{-1}\left(m_{t}\right) * u_{0} \in \mathcal{S}^{\prime}\left(\mathbb{R}^{2}\right) .
$$

For $\delta_{1}, \delta_{2}>0$ let us define $m_{t}^{\delta_{1}, \delta_{2}}(\xi, \eta):=e^{-\delta_{1} \xi^{2}-\delta_{2} \eta^{2}} m_{t}(\xi, \eta)$. Then by the theorem of dominated convergence we have that $\lim _{\delta_{1}, \delta_{2} \rightarrow 0+} m_{t}^{\delta_{1}, \delta_{2}}=m_{t}$ in $\mathcal{S}^{\prime}\left(\mathbb{R}^{2}\right)$. Therefore we have

$$
\left|D_{x}\right|^{-\theta+i \kappa} U_{\alpha}(t) u_{0}=\lim _{\delta_{1}, \delta_{2} \rightarrow 0+} \mathcal{F}^{-1}\left(m_{t}^{\delta_{1}, \delta_{2}}\right) * u_{0} .
$$

Now we have

$$
\begin{aligned}
\mathcal{F}^{-1}\left(m_{t}^{\delta_{1}, \delta_{2}}\right)(x, y) & =c \int_{\mathbb{R}^{2}} e^{i(x \xi+y \eta)} m_{t}^{\delta_{1}, \delta_{2}}(\xi, \eta) d \xi d \eta \\
& =c \int_{\mathbb{R}}|\xi|^{-\theta+i \kappa} e^{-\delta_{1} \xi^{2}+i\left(x \xi+t \xi|\xi|^{\alpha}\right)}\left(\int_{\mathbb{R}} e^{i y \eta} e^{-\left(\delta_{2}+\frac{i t}{\xi}\right) \eta^{2}} d \eta\right) d \xi \\
& =c \int_{\mathbb{R}}|\xi|^{-\theta+i \kappa} e^{-\delta_{1} \xi^{2}+i x \xi+i t \xi|\xi|^{\alpha}}\left(\delta_{2}+\frac{i t}{\xi}\right)^{-\frac{1}{2}} e^{-\frac{1}{4}\left(\delta_{2}+\frac{i t}{\xi}\right)^{-1} y^{2}} d \xi
\end{aligned}
$$

Now again by the theorem of dominated convergence we can take the limit $\delta_{2} \rightarrow 0+$ in this last expression and get

$$
\mathcal{F}^{-1}\left(m_{t}\right)=c|t|^{-\frac{1}{2}} \lim _{\delta_{1} \rightarrow 0+} \int_{\mathbb{R}}|\xi|^{\frac{1}{2}-\theta+i \kappa} e^{-\delta_{1} \xi^{2}+i\left(x \xi+t \xi|\xi|^{\alpha}-\operatorname{sign}\left(\frac{\xi}{t}\right) \frac{\pi}{4}\right)} e^{\frac{i \xi}{4 t} y^{2}} d \xi .
$$

Now for $\xi \neq 0$ we set $\psi(\xi):=e^{-\delta_{1} \xi^{2}-i \operatorname{sign}\left(\frac{\xi}{t}\right) \frac{\pi}{4}}$ and $\phi(\xi):=\xi|\xi|^{\alpha}$. Then with our choice of $\theta$ we have $\left|\phi^{\prime \prime}(\xi)\right| \sim|\xi|^{1-2 \theta}$, and we can use Corollary 2.9 of [9] to see that

$$
\left.\left.\left|\int_{\mathbb{R}}\right| \xi\right|^{\frac{1}{2}-\theta+i \kappa} e^{-\delta_{1} \xi^{2}+i\left(x \xi+t \xi|\xi|^{\alpha}-\operatorname{sign}\left(\frac{\xi}{t}\right) \frac{\pi}{4}\right)} e^{\frac{i \xi}{4 t} y^{2}} d \xi|\lesssim\langle\kappa\rangle| t\right|^{-\frac{1}{2}}
$$


where the implicit constant does not depend on $\delta_{1}>0$. Therefore we get that $\mathcal{F}^{-1}\left(m_{t}\right) \in L^{\infty}\left(\mathbb{R}^{2}\right)$ and $\left\|\mathcal{F}^{-1}\left(m_{t}\right)\right\|_{L^{\infty}\left(\mathbb{R}^{2}\right)} \leq C\langle\kappa\rangle|t|^{-1}$. It follows that we have the decay estimate

$$
\left\|\left|D_{x}\right|^{-\theta+i \kappa} U_{\alpha}(t) u_{0}\right\|_{L^{\infty}} \leq C\langle\kappa\rangle|t|^{-1}\left\|u_{0}\right\|_{L^{1}}
$$

for all $u_{0} \in \mathcal{S}\left(\mathbb{R}^{2}\right)$ and then by continuity also for all $u_{0} \in L^{1}\left(\mathbb{R}^{2}\right)$. By Plancherel we also have that $\left\|\left|D_{x}\right|^{i \kappa} U_{\alpha}(t) u_{0}\right\|_{L^{2}}=\left\|u_{0}\right\|_{L^{2}}$. Now using the interpolation theorem of Stein we get for every $2 \leq r \leq \infty$ that

$$
\left\|\left|D_{x}\right|^{\left(\frac{2}{r}-1\right) \theta} U_{\alpha}(t) u_{0}\right\|_{L^{r}} \leq C|t|^{\frac{2}{r}-1}\left\|u_{0}\right\|_{L^{r^{\prime}}} .
$$

Now (3.1) follows from (3.2) by well-known methods. (See for example 9].)

From this linear version of Strichartz estimates we can deduce the following bilinear version.

Corollary 3.2. For $b>\frac{1}{2}$ we have

$$
\left\|u_{1} u_{2}\right\|_{L^{2}} \lesssim\left\|\left|D_{x}\right|^{\frac{1}{4}-\frac{\alpha}{8}} u_{1}\right\|_{X_{0}^{0, b}}\left\|\left|D_{x}\right|^{\frac{1}{4}-\frac{\alpha}{8}} u_{2}\right\|_{X_{0}^{0, b}}
$$

Furthermore we have

$$
\begin{array}{r}
\left|\int_{\mathbb{R}^{6}} \frac{\left|\xi_{1}\right|^{-\frac{1}{4}+\frac{\alpha}{8}}\left|\xi-\xi_{1}\right|^{-\frac{1}{4}+\frac{\alpha}{8}}}{\left\langle\lambda_{1}\right\rangle^{b}\left\langle\lambda_{2}\right\rangle^{b}} f_{1}\left(\mu_{1}\right) f_{2}\left(\mu-\mu_{1}\right) f_{3}(\mu) d \mu_{1} d \mu\right| \lesssim \prod_{i=1}^{3}\left\|f_{i}\right\|_{L^{2}}, \\
\left|\int_{\mathbb{R}^{6}} \frac{\left|\xi_{1}\right|^{-\frac{1}{4}+\frac{\alpha}{8}}|\xi|^{-\frac{1}{4}+\frac{\alpha}{8}}}{\left\langle\lambda_{1}\right\rangle^{b}\langle\lambda\rangle^{b}} f_{1}\left(\mu_{1}\right) f_{2}\left(\mu-\mu_{1}\right) f_{3}(\mu) d \mu_{1} d \mu\right| \lesssim \prod_{i=1}^{3}\left\|f_{i}\right\|_{L^{2}}, \\
\left|\int_{\mathbb{R}^{6}} \frac{|\xi|^{-\frac{1}{4}+\frac{\alpha}{8}}\left|\xi-\xi_{1}\right|^{-\frac{1}{4}+\frac{\alpha}{8}}}{\langle\lambda\rangle^{b}\left\langle\lambda_{2}\right\rangle^{b}} f_{1}\left(\mu_{1}\right) f_{2}\left(\mu-\mu_{1}\right) f_{3}(\mu) d \mu_{1} d \mu\right| \lesssim \prod_{i=1}^{3}\left\|f_{i}\right\|_{L^{2}} .
\end{array}
$$

Proof. Setting $r=q=4$ in (3.1) we get $\left\|\left|D_{x}\right|^{-\left(\frac{1}{4}-\frac{\alpha}{8}\right)} U_{\alpha}(t) u_{0}\right\|_{L_{t x y}^{4}} \lesssim\left\|u_{0}\right\|_{L_{x y}^{2}}$. Using [4, Lemme 3.3, it follows that $\left\|\left|D_{x}\right|^{-\left(\frac{1}{4}-\frac{\alpha}{8}\right)} u\right\|_{L_{t x y}^{4}} \lesssim\|u\|_{X_{0}^{0, b}}$ or equivalently $\|u\|_{L_{t x y}^{4}} \lesssim\left\|\left|D_{x}\right|^{\frac{1}{4}-\frac{\alpha}{8}} u\right\|_{X_{0}^{0, b}}$. Now (3.3) follows by combining this estimate with Hölder's inequality. Setting $f_{i}(\mu):=|\xi|^{\frac{1}{4}-\frac{\alpha}{8}}\langle\lambda\rangle^{b} \widehat{u}_{i}(\mu)$ for $i=1,2$ and using duality, we see that (3.3) is equivalent to (3.4). By suitable changes of variables we also get (3.5) and (3.6).

For the part of the product $u_{1} u_{2}$, where the $\xi$-frequency of the first factor is significantly smaller than the $\xi$-frequency of the second factor, we can improve this bilinear Strichartz estimate. To formulate this improvement let us define for $c>0$ the following operator:

$$
\mathcal{F} P_{c}\left(u_{1}, u_{2}\right)(\mu):=\int_{\mathbb{R}^{3}} \chi_{\left|\xi_{1}\right| \leq c\left|\xi-\xi_{1}\right|}\left(\mu, \mu_{1}\right) \mathcal{F} u_{1}\left(\mu_{1}\right) \mathcal{F} u_{2}\left(\mu-\mu_{1}\right) d \mu_{1} .
$$

We have the following refined bilinear Strichartz estimate which for the case $\alpha=2$ was already implicitly used in [18, 15, 19, 16, 17, 6].

Theorem 3.3. For $b>\frac{1}{2}$ we have

$$
\left\|P_{\frac{1}{3}}\left(u_{1}, u_{2}\right)\right\|_{L^{2}} \lesssim\left\|\left|D_{x}\right|^{\frac{1}{2}} u_{1}\right\|_{X_{0}^{0, b}}\left\|\left|D_{x}\right|^{-\frac{\alpha}{4}} u_{2}\right\|_{X_{0}^{0, b}}
$$


For the proof of the theorem we need the following lemma.

Lemma 3.4. For $\alpha>0$ set $\phi_{\alpha}(\xi):=\xi|\xi|^{\alpha}$ and

$$
r_{\alpha}\left(\xi, \xi_{1}\right):=\phi_{\alpha}(\xi)-\phi_{\alpha}\left(\xi_{1}\right)-\phi_{\alpha}\left(\xi-\xi_{1}\right), \quad \xi, \xi_{1} \in \mathbb{R} .
$$

We then have for every $\xi, \xi_{1} \in \mathbb{R}$

$$
\frac{\alpha}{2^{\alpha}}\left|\xi_{\min }\right|\left|\xi_{\max }\right|^{\alpha} \leq\left|r_{\alpha}\left(\xi, \xi_{1}\right)\right| \leq\left(\alpha+1+\frac{1}{2^{\alpha}}\right)\left|\xi_{\min }\right|\left|\xi_{\max }\right|^{\alpha} .
$$

Proof of Lemma 3.4. Suppose first that $\left|\xi_{\min }\right|=\left|\xi_{1}\right|$. Then we have

$$
\left|\phi_{\alpha}\left(\xi_{1}\right)\right|=\left|\xi_{\min }\right|^{\alpha+1} \leq \frac{1}{2^{\alpha}}\left|\xi_{\min }\right|\left|\xi_{\max }\right|^{\alpha}
$$

because $\left|\xi_{\min }\right| \leq \frac{1}{2}\left|\xi_{\max }\right|$ and

$$
\left|\phi_{\alpha}(\xi)-\phi_{\alpha}\left(\xi-\xi_{1}\right)\right|=\left|\phi_{\alpha}^{\prime}\left(\xi-\theta \xi_{1}\right)\right|\left|\xi_{1}\right|=(\alpha+1)\left|\xi-\theta \xi_{1}\right|^{\alpha}\left|\xi_{\min }\right|
$$

for some $\theta \in[0,1]$. As we have $\left|\xi_{1}\right| \leq|\xi|$ it follows that

$$
\min _{\theta \in[0,1]}\left|\xi-\theta \xi_{1}\right|=\min \left\{|\xi|,\left|\xi-\xi_{1}\right|\right\}=\left|\xi_{\text {med }}\right| \geq \frac{1}{2}\left|\xi_{\max }\right|
$$

and

$$
\max _{\theta \in[0,1]}\left|\xi-\theta \xi_{1}\right|=\max \left\{|\xi|,\left|\xi-\xi_{1}\right|\right\}=\left|\xi_{\max }\right| .
$$

Putting these estimates together we get

$$
\begin{aligned}
\left|r_{\alpha}\left(\xi, \xi_{1}\right)\right| & \geq\left|\phi_{\alpha}(\xi)-\phi_{\alpha}\left(\xi-\xi_{1}\right)\right|-\left|\phi_{\alpha}\left(\xi_{1}\right)\right| \\
& \geq(\alpha+1) \frac{1}{2^{\alpha}}\left|\xi_{\max }\right|^{\alpha}\left|\xi_{\min }\right|-\frac{1}{2^{\alpha}}\left|\xi_{\min }\right|\left|\xi_{\max }\right|^{\alpha} \\
& =\frac{\alpha}{2^{\alpha}}\left|\xi_{\min }\right|\left|\xi_{\max }\right|^{\alpha}
\end{aligned}
$$

and

$$
\begin{aligned}
\left|r_{\alpha}\left(\xi, \xi_{1}\right)\right| & \leq\left|\phi_{\alpha}(\xi)-\phi_{\alpha}\left(\xi-\xi_{1}\right)\right|+\left|\phi_{\alpha}\left(\xi_{1}\right)\right| \\
& \leq(\alpha+1)\left|\xi_{\max }\right|^{\alpha}\left|\xi_{\min }\right|+\frac{1}{2^{\alpha}}\left|\xi_{\min }\right|\left|\xi_{\max }\right|^{\alpha} \\
& =\left(\alpha+1+\frac{1}{2^{\alpha}}\right)\left|\xi_{\min }\right|\left|\xi_{\max }\right|^{\alpha}
\end{aligned}
$$

which proves (3.10) in the case $\left|\xi_{\min }\right|=\left|\xi_{1}\right|$. Noting that $r_{\alpha}\left(\xi, \xi_{1}\right)=r_{\alpha}\left(\xi, \xi-\xi_{1}\right)=$ $-r_{\alpha}\left(\xi-\xi_{1}, \xi\right)$ we see that we also get (3.10) in the other cases.

Proof of Theorem 3.3, Let $f_{1}(\mu):=|\xi|^{\frac{1}{2}}\langle\lambda\rangle^{b} \widehat{u_{1}}(\mu)$ and $f_{2}(\mu):=|\xi|^{-\frac{\alpha}{4}}\langle\lambda\rangle^{b} \widehat{u_{2}}(\mu)$. We have to show that

$$
\left\|\int_{\mathbb{R}^{3}} \chi_{\left|\xi_{1}\right| \leq \frac{1}{3}\left|\xi-\xi_{1}\right|} \frac{\left|\xi_{1}\right|^{-\frac{1}{2}}\left|\xi-\xi_{1}\right|^{\frac{\alpha}{4}}}{\left\langle\lambda_{1}\right\rangle^{b}\left\langle\lambda_{2}\right\rangle^{b}} f_{1}\left(\mu_{1}\right) f_{2}\left(\mu-\mu_{1}\right) d \mu_{1}\right\|_{L_{\mu}^{2}} \lesssim\left\|f_{1}\right\|_{L^{2}}\left\|f_{2}\right\|_{L^{2}}
$$

which by duality is equivalent to

$$
\left|\int_{\mathbb{R}^{6}} \chi_{\left|\xi_{1}\right| \leq \frac{1}{3}\left|\xi-\xi_{1}\right|} \frac{\left|\xi_{1}\right|^{-\frac{1}{2}}\left|\xi-\xi_{1}\right|^{\frac{\alpha}{4}}}{\left\langle\lambda_{1}\right\rangle^{b}\left\langle\lambda_{2}\right\rangle^{b}} f_{1}\left(\mu_{1}\right) f_{2}\left(\mu-\mu_{1}\right) f_{3}(\mu) d \mu_{1} d \mu\right| \lesssim \prod_{i=1}^{3}\left\|f_{i}\right\|_{L^{2}}
$$


By use of the Cauchy-Schwarz inequality it suffices to show that $\sup _{\mu} I(\mu)^{\frac{1}{2}}<\infty$ where

$$
I(\mu):=\int_{\mathbb{R}^{3}} \chi_{\left|\xi_{1}\right| \leq \frac{1}{3}\left|\xi-\xi_{1}\right|} \frac{\left|\xi_{1}\right|^{-1}\left|\xi-\xi_{1}\right|^{\frac{\alpha}{2}}}{\left\langle\lambda_{1}\right\rangle^{2 b}\left\langle\lambda_{2}\right\rangle^{2 b}} d \mu_{1} .
$$

For fixed $\mu$ we now use the change of variables $T: \mu_{1} \mapsto\left(\nu, \lambda_{1}, \lambda_{2}\right)$ where

$$
\nu\left(\mu_{1}\right):=r_{\alpha}\left(\xi, \xi_{1}\right)=\xi|\xi|^{\alpha}-\xi_{1}\left|\xi_{1}\right|^{\alpha}-\left(\xi-\xi_{1}\right)\left|\xi-\xi_{1}\right|^{\alpha} .
$$

Let us also recall the definition of $\lambda_{1}$ and $\lambda_{2}$ :

$$
\begin{aligned}
& \lambda_{1}\left(\mu_{1}\right)=\tau_{1}-\xi_{1}\left|\xi_{1}\right|^{\alpha}+\frac{\eta_{1}^{2}}{\xi_{1}} \\
& \lambda_{2}\left(\mu_{1}\right)=\tau-\tau_{1}-\left(\xi-\xi_{1}\right)\left|\xi-\xi_{1}\right|^{\alpha}+\frac{\left(\eta-\eta_{1}\right)^{2}}{\xi-\xi_{1}} .
\end{aligned}
$$

Observe that

$$
\lambda_{1}+\lambda_{2}-\lambda=\nu+\frac{\left(\xi \eta_{1}-\eta \xi_{1}\right)^{2}}{\xi \xi_{1}\left(\xi-\xi_{1}\right)} .
$$

Therefore we have

$$
\left|\partial_{\eta_{1}}\left(\lambda_{1}+\lambda_{2}\right)\right|=2\left|\xi \frac{\xi \eta_{1}-\eta \xi_{1}}{\xi \xi_{1}\left(\xi-\xi_{1}\right)}\right|=2 \frac{|\xi|^{\frac{1}{2}}\left|\lambda_{1}+\lambda_{2}-\lambda-\nu\right|^{\frac{1}{2}}}{\left|\xi_{1}\right|^{\frac{1}{2}}\left|\xi-\xi_{1}\right|^{\frac{1}{2}}} .
$$

Furthermore we have $\partial_{\xi_{1}} \nu=(\alpha+1)\left(\left|\xi-\xi_{1}\right|^{\alpha}-\left|\xi_{1}\right|^{\alpha}\right)$. As we only consider the region where $\left|\xi_{1}\right| \leq \frac{1}{3}\left|\xi-\xi_{1}\right|$, i.e. $\left|\xi_{1}\right|=\left|\xi_{\min }\right|$ and $\left|\xi-\xi_{1}\right| \sim\left|\xi_{\max }\right|$, we have by (3.10) that $|\nu| \sim\left|\xi_{1}\right|\left|\xi-\xi_{1}\right|^{\alpha}$. We also have $\left|\partial_{\xi_{1}} \nu\right| \gtrsim\left|\xi-\xi_{1}\right|^{\alpha}$ in this region. Therefore we have that

$$
\begin{aligned}
\left|D_{\mu_{1}} T\right| & =\left|\partial_{\xi_{1}} \nu\right|\left|\partial_{\eta_{1}} \lambda_{1}+\partial_{\eta_{1}} \lambda_{2}\right| \gtrsim\left|\xi_{1}\right|^{-\frac{1}{2}}\left|\xi-\xi_{1}\right|^{\alpha}\left|\lambda_{1}+\lambda_{2}-\lambda-\nu\right|^{\frac{1}{2}} \\
& \gtrsim\left|\xi_{1}\right|^{-1}\left|\xi-\xi_{1}\right|^{\frac{\alpha}{2}}|\nu|^{\frac{1}{2}}\left|\lambda_{1}+\lambda_{2}-\lambda-\nu\right|^{\frac{1}{2}} .
\end{aligned}
$$

Let us notice that it is possible to divide the region of integration into a finite number of open subsets $U_{i}$ such that $T$ is an injective $C^{1}$-function in $U_{i}$ with nonvanishing Jacobian. As we are in the KP-II-case both terms on the right hand side of (3.12) have the same sign which implies that $|\nu| \leq\left|\lambda_{1}+\lambda_{2}-\lambda\right|$. So performing the change of variables and using the elementary inequality

$$
\int_{-K}^{K} \frac{d \nu}{|\nu|^{\frac{1}{2}}|a-\nu|^{\frac{1}{2}}} \lesssim \frac{K^{\frac{1}{2}}}{|a|^{\frac{1}{2}}}, \quad a \neq 0,
$$

we get

$$
I(\mu) \lesssim \int_{\mathbb{R}^{3}} \frac{\chi_{|\nu| \leq\left|\lambda_{1}+\lambda_{2}-\lambda\right|} d \nu d \lambda_{1} d \lambda_{2}}{\left\langle\lambda_{1}\right\rangle^{2 b}\left\langle\lambda_{2}\right\rangle^{2 b}|\nu|^{\frac{1}{2}}\left|\lambda_{1}+\lambda_{2}-\lambda-\nu\right|^{\frac{1}{2}}} \lesssim \int_{\mathbb{R}^{2}} \frac{d \lambda_{1} d \lambda_{2}}{\left\langle\lambda_{1}\right\rangle^{2 b}\left\langle\lambda_{2}\right\rangle^{2 b}} \lesssim 1
$$

In fact we also get (3.11) without the cut-off function $\chi_{\left|\xi_{1}\right| \leq \frac{1}{3}\left|\xi-\xi_{1}\right|}$ and we also get dual versions of (3.11). 
Proposition 3.5. For $b>\frac{1}{2}$ we have

$$
\begin{aligned}
\left|\int_{\mathbb{R}^{6}} \frac{\left|\xi_{1}\right|^{-\frac{1}{2}}\left|\xi-\xi_{1}\right|^{\frac{\alpha}{4}}}{\left\langle\lambda_{1}\right\rangle^{b}\left\langle\lambda_{2}\right\rangle^{b}} f_{1}\left(\mu_{1}\right) f_{2}\left(\mu-\mu_{1}\right) f_{3}(\mu) d \mu_{1} d \mu\right| & \lesssim \prod_{i=1}^{3}\left\|f_{i}\right\|_{L^{2}}, \\
\left|\int_{\mathbb{R}^{6}} \frac{\left|\xi_{1}\right|^{-\frac{1}{2}}|\xi|^{\frac{\alpha}{4}}}{\left\langle\lambda_{1}\right\rangle^{b}\langle\lambda\rangle^{b}} f_{1}\left(\mu_{1}\right) f_{2}\left(\mu-\mu_{1}\right) f_{3}(\mu) d \mu_{1} d \mu\right| & \lesssim \prod_{i=1}^{3}\left\|f_{i}\right\|_{L^{2}}, \\
\left|\int_{\mathbb{R}^{6}} \frac{|\xi|^{-\frac{1}{2}}\left|\xi-\xi_{1}\right|^{\frac{\alpha}{4}}}{\langle\lambda\rangle^{b}\left\langle\lambda_{2}\right\rangle^{b}} f_{1}\left(\mu_{1}\right) f_{2}\left(\mu-\mu_{1}\right) f_{3}(\mu) d \mu_{1} d \mu\right| & \lesssim \prod_{i=1}^{3}\left\|f_{i}\right\|_{L^{2}}, \\
\left|\int_{\mathbb{R}^{6}} \frac{|\xi|^{-\frac{1}{2}}\left|\xi_{1}\right|^{\frac{\alpha}{4}}}{\langle\lambda\rangle^{b}\left\langle\lambda_{1}\right\rangle^{b}} f_{1}\left(\mu_{1}\right) f_{2}\left(\mu-\mu_{1}\right) f_{3}(\mu) d \mu_{1} d \mu\right| & \lesssim \prod_{i=1}^{3}\left\|f_{i}\right\|_{L^{2}} .
\end{aligned}
$$

Proof. In the region where $\left|\xi_{1}\right| \leq \frac{1}{3}\left|\xi-\xi_{1}\right|$ the estimate (3.13) follows from the estimate (3.11). In the region where $\left|\xi_{1}\right|>\frac{1}{3}\left|\xi-\xi_{1}\right|$ we have that $\left|\xi_{1}\right|^{-\frac{1}{2}}\left|\xi-\xi_{1}\right|^{\frac{\alpha}{4}} \lesssim$ $\left|\xi_{1}\right|^{-\frac{1}{4}+\frac{\alpha}{8}}\left|\xi-\xi_{1}\right|^{-\frac{1}{4}+\frac{\alpha}{8}}$, and so the estimate in this region follows from the bilinear Strichartz estimate (3.4). As in the case of the bilinear Strichartz estimates we now get the dual versions (3.14), (3.15) and (3.16) by an appropriate change of variables.

\section{The Main BiLinear estimate}

In the following formulation and proof of the crucial bilinear estimate needed to prove Theorem 1.2 we will only consider the case $s_{2}=0$ (and write $s$ for $s_{1}$ ) to simplify the presentation. Note that the case $s_{2}>0$ follows from this special case; as in the general case we only get an extra term $\frac{\langle\eta\rangle^{s_{2}}}{\left\langle\eta_{1}\right\rangle^{s_{2}}\left\langle\eta-\eta_{1}\right\rangle^{s_{2}}}$ in the integral inequalities that we have to prove (see (4.6) ). But this term is always bounded above for $s_{2} \geq 0$.

Theorem 4.1. For $\frac{4}{3}<\alpha \leq 6$ and $s>\max \left(1-\frac{3}{4} \alpha, \frac{1}{4}-\frac{3}{8} \alpha\right)$ let us choose $0<\varepsilon \leq \frac{1}{16}$ such that

$$
\varepsilon \leq \min \left(\frac{1}{\alpha+1}\left(s+\frac{3}{4} \alpha-1\right), \frac{1}{\alpha+1}\left(\frac{3}{4} \alpha-1\right), \frac{1}{\alpha}\left(2 s+\frac{3}{4} \alpha-\frac{1}{2}\right)\right)
$$

and set $b:=\frac{1}{2}+\frac{\varepsilon}{2}, b^{\prime}=-\frac{1}{2}+\varepsilon, b_{1}:=\max \left(\frac{3}{2 \alpha}-\frac{3}{4}+\varepsilon, 0\right)$ and $\sigma=\frac{1}{2}+b_{1}$. Let us define $X=X_{1}+X_{2}$ and $\tilde{X}=\tilde{X}_{1}+\tilde{X}_{2}$ where

$$
\begin{array}{cc}
X_{1}:=X_{0}^{s, b-b^{\prime}}, & X_{2}:=X_{\sigma}^{s, b} \cap X_{\sigma}^{s-(\alpha+1) b_{1}, b+b_{1}}, \\
\tilde{X}_{1}:=X_{0}^{s, 0}, & \tilde{X}_{2}:=X_{\sigma}^{s, b^{\prime}} \cap X_{\sigma}^{s-(\alpha+1) b_{1}, b^{\prime}+b_{1}} .
\end{array}
$$

We then have

$$
\left\|\partial_{x}\left(u_{1} u_{2}\right)\right\|_{\tilde{X}} \leq C\left\|u_{1}\right\|_{X}\left\|u_{2}\right\|_{X} .
$$

Remark 4.2. The spaces $X$ and $\tilde{X}$ defined in Theorem 4.1 are built by taking sums and intersections of the Bourgain type spaces of Section 2. Therefore it is easy to see that they also satisfy the linear estimates of Propositions 2.5] and 2.6. i.e.

$$
\left\|\psi U_{\alpha}(t) u_{0}\right\|_{X} \lesssim\left\|u_{0}\right\|_{H^{s, 0}\left(\mathbb{R}^{2}\right)}
$$

and

$$
\left\|\psi_{T} \int_{0}^{t} U\left(t-t^{\prime}\right) F\left(t^{\prime}\right) d t^{\prime}\right\|_{X} \lesssim T^{1-\left(b-b^{\prime}\right)}\|F\|_{\tilde{X}}
$$


Remark 4.3. The sum structure of the spaces $X$ and $\tilde{X}$ is the essential ingredient to use the additional weight $\left(\frac{\langle\xi\rangle}{|\xi|}\right)^{\sigma}$, which is incorporated in the definition of $X_{2}$ and $\tilde{X}_{2}$ (see (2.2) ), to lower the $x$-regularity $s$ in the bilinear estimate without imposing a low frequency condition on the initial data. Therefore, in the case $\alpha=2$ of the Kadomtsev-Petviashvili II equation we are able to show the local well-posedness for all $s>-\frac{1}{2}$ without a low frequency condition on the initial data, whereas the counterexamples in [17] show that it is not possible to get the bilinear estimate for $-\frac{1}{2}<s<-\frac{1}{3}$ and $\sigma=0$.

Remark 4.4. In the case $\alpha=4$ of the fifth order KP II equation it is possible to get the bilinear estimate (4.1) in the spaces $X=X_{0}^{s, b}$ and $\tilde{X}=X_{0}^{s, b^{\prime}}$ (i.e. choosing $b_{1}=0$ and $\left.\sigma=0\right)$ as is shown in [7]. More generally, this is true for all $\alpha>\frac{5}{2}$ which can be seen by refining the estimate of Lemma 4.9 by an additional dyadic decomposition and interpolation argument as used in [17, pp. 89-92.

While deriving the bilinear estimate (4.1) we can, in most of the cases, take $u_{1}$ and $u_{2}$ in the simpler space $X_{0}^{s, b}$ instead of $X$ for the calculations and then use the following simple embedding property.

Lemma 4.5. For $X$ defined as in Theorem 4.1 we have $X \hookrightarrow X_{0}^{s, b}$. More precisely, we have the estimate

$$
\|u\|_{X_{0}^{s, b}} \leq\|u\|_{X}
$$

Proof. Because of $\sigma \geq 0$ we have that $\left(\frac{\langle\xi\rangle}{|\xi|}\right)^{\sigma} \geq 1$. Then by definition (2.2) of the $X_{\sigma}^{s, b}$-norm we have $\|u\|_{X_{0}^{s, b}} \leq\|u\|_{X_{\sigma}^{s, b}} \leq\|u\|_{X_{2}}$. As $b^{\prime} \leq 0$, we have $\langle\lambda\rangle^{-b^{\prime}} \geq 1$. Again by definition (2.2) it follows that $\|u\|_{X_{0}^{s, b}} \leq\|u\|_{X_{0}^{s, b-b^{\prime}}}=\|u\|_{X_{1}}$. The norm in $X$ is given by

$$
\|u\|_{X}=\inf \left\{\|v\|_{X_{1}}+\|w\|_{X_{2}} \mid u=v+w, v \in X_{1}, w \in X_{2}\right\} .
$$

If $u \in X$ and $u=v+w$ with $v \in X_{1}$ and $w \in X_{2}$, we get $\|u\|_{X_{0}^{s, b}} \leq\|v\|_{X_{1}}+\|w\|_{X_{2}}$. If we now take the infimum on the right hand side of this inequality over all possible decompositions of $u$ of the form $u=v+w$ with $v \in X_{1}$ and $w \in X_{2}$, we get (4.4).

To prove Theorem 4.1 we will split the non-linear term $\partial_{x}\left(u_{1} u_{2}\right)$ into various pieces and give estimates in appropriate $X_{\sigma}^{s, b}$-spaces for each of these pieces (see Lemmas 4.6 4.12). We will then combine these estimates to prove (4.1). First of all, with $P_{c}$ defined as in (3.7), we can write

$$
\partial_{x}\left(u_{1} u_{2}\right)=\partial_{x} P_{1}\left(u_{1}, u_{2}\right)+\partial_{x} P_{1}\left(u_{2}, u_{1}\right) .
$$

As the main bilinear estimate (4.1) is symmetric in $u_{1}$ and $u_{2}$, it suffices to prove it only for $\partial_{x} P_{1}\left(u_{1}, u_{2}\right)$. This expression can be decomposed further into

$$
\partial_{x} P_{1}\left(u_{1} u_{2}\right)=Q_{00}\left(u_{1}, u_{2}\right)+\sum_{i=1}^{2} \sum_{j=0}^{2} Q_{i j}\left(u_{1}, u_{2}\right) .
$$

The operators $Q_{i j}$ are defined by

$$
\mathcal{F} Q_{i j}\left(u_{1}, u_{2}\right)(\mu)=i \xi \int_{\mathbb{R}^{3}} \chi_{A_{i j}}\left(\mu_{1}, \mu\right) \mathcal{F} u_{1}\left(\mu_{1}\right) \mathcal{F} u_{2}\left(\mu-\mu_{1}\right) d \mu_{1},
$$


where $A_{00}:=\left\{\left(\mu_{1}, \mu\right) \in \mathbb{R}^{6}|| \xi_{1}|\leq| \xi-\xi_{1} \mid \leq 1\right\}$ and $A_{i j}:=\Xi_{i} \cap \Lambda_{j}$ for $1 \leq i \leq 2$, $0 \leq j \leq 2$ with

$$
\begin{aligned}
& \Xi_{1}=\left\{\left(\mu_{1}, \mu\right) \in \mathbb{R}^{6}|| \xi_{1}\left|\leq \frac{1}{3}\right| \xi-\xi_{1}|,| \xi-\xi_{1} \mid \geq 1\right\}, \\
& \Xi_{2}=\left\{\left(\mu_{1}, \mu\right) \in \mathbb{R}^{6}\left|\frac{1}{3}\right| \xi-\xi_{1}|\leq| \xi_{1}|\leq| \xi-\xi_{1}|,| \xi-\xi_{1} \mid \geq 1\right\}, \\
& \Lambda_{0}=\left\{\left(\mu_{1}, \mu\right) \in \mathbb{R}^{6}|| \lambda|=| \lambda_{\max } \mid\right\}, \\
& \Lambda_{j}=\left\{\left(\mu_{1}, \mu\right) \in \mathbb{R}^{6}|| \lambda_{j}|=| \lambda_{\max } \mid\right\} \quad(j=1,2) .
\end{aligned}
$$

Let us explain the meaning of the regions $\Xi_{1}$ and $\Xi_{2}$. In $\Xi_{1}$ we have that $2 \leq$ $2\left|\xi-\xi_{1}\right| \leq 3|\xi| \leq 4\left|\xi-\xi_{1}\right|$, i.e. $\xi$ and $\xi-\xi_{1}$ are comparable in size and are both bounded away from zero, whereas $\xi_{1}$ is the smallest of the frequencies dual to the $x$-variable. In $\Xi_{2}$ we have that $\xi_{1}$ and $\xi-\xi_{1}$ are comparable in size and are both bounded away from zero, whereas $\xi$ may be small here. For each of the operators $Q_{i j}$ we will now show estimates of the form

$$
\left\|Q_{i j}\left(u_{1}, u_{2}\right)\right\|_{X_{\sigma^{\prime}}^{s^{\prime}, b^{\prime}}} \lesssim\left\|u_{1}\right\|_{X_{\sigma_{1}}^{s_{1}, b_{1}}}\left\|u_{2}\right\|_{X_{\sigma_{2}}^{s_{2}, b_{2}}} .
$$

By definition (2.2) of the $X_{\sigma}^{s, b}$-norm and setting $f_{l}(\mu):=|\xi|^{-\sigma}\langle\xi\rangle^{s_{l}+\sigma}\langle\lambda\rangle^{b_{l}} \widehat{u}_{l}(\mu)$ this is equivalent to

$$
\left\|\frac{|\xi|\langle\xi\rangle^{s^{\prime}+\sigma^{\prime}}}{|\xi|^{\sigma^{\prime}}\langle\lambda\rangle^{-b^{\prime}}} \int_{\mathbb{R}^{3}} \chi_{A_{i j}}\left(\mu_{1}, \mu\right) \frac{\left|\xi_{1}\right|^{\sigma_{1}} f_{1}\left(\mu_{1}\right)\left|\xi-\xi_{1}\right|^{\sigma_{2}} f_{2}\left(\mu-\mu_{1}\right) d \mu_{1}}{\left\langle\xi_{1}\right\rangle^{s_{1}+\sigma_{1}}\left\langle\lambda_{1}\right\rangle^{b_{1}}\left\langle\xi-\xi_{1}\right\rangle^{s_{2}+\sigma_{2}}\left\langle\lambda_{2}\right\rangle^{b_{2}}}\right\|_{L_{\mu}^{2}} \lesssim\left\|f_{1}\right\|_{L^{2}}\left\|f_{2}\right\|_{L^{2}} .
$$

Using duality this estimate is equivalent to

$$
\left|\int_{A_{i j}} \frac{|\xi|\langle\xi\rangle^{s^{\prime}+\sigma^{\prime}}\left|\xi_{1}\right|^{\sigma_{1}}\left|\xi-\xi_{1}\right|^{\sigma_{2}} f_{1}\left(\mu_{1}\right) f_{2}\left(\mu-\mu_{1}\right) f_{3}(\mu)}{|\xi|^{\sigma^{\prime}}\left\langle\xi_{1}\right\rangle^{s_{1}+\sigma_{1}}\left\langle\xi-\xi_{1}\right\rangle^{s_{2}+\sigma_{2}}\langle\lambda\rangle^{-b^{\prime}}\left\langle\lambda_{1}\right\rangle^{b}\left\langle\lambda_{2}\right\rangle^{b}} d \mu d \mu_{1}\right| \lesssim \prod_{i=1}^{3}\left\|f_{i}\right\|_{L^{2}} .
$$

The main ingredients that we use in the proof of these estimates are the bilinear Strichartz estimates of Corollary 3.2 and Theorem 3.3 and the use of the "resonance identity" (3.12). We already noted that the two terms on the right hand side of (3.12) have the same sign. Therefore we have

$$
\left|\lambda_{\max }\right| \geq \frac{1}{3}\left|\lambda_{1}+\lambda_{2}-\lambda\right| \geq \frac{1}{3}|\nu| \geq \frac{\alpha}{3 \cdot 2^{\alpha}}\left|\xi_{\min }\right|\left|\xi_{\max }\right|^{\alpha},
$$

where for the last inequality we used (3.10).

Lemma 4.6. We have that

$$
\left\|Q_{00}\left(u_{1}, u_{2}\right)\right\|_{X_{0}^{s, 0}} \lesssim\left\|u_{1}\right\|_{X_{0}^{s, b}}\left\|u_{2}\right\|_{X_{0}^{s, b}}
$$

provided that $b>\frac{1}{2}, \alpha \leq 6$ and $s \in \mathbb{R}$.

Proof. We have to prove that

$$
\left|\int_{A_{00}} k_{00}\left(\mu_{1}, \mu\right) \frac{\left|\xi_{1}\right|^{-\frac{1}{2}}\left|\xi-\xi_{1}\right|^{\frac{\alpha}{4}}}{\left\langle\lambda_{1}\right\rangle^{b}\left\langle\lambda_{2}\right\rangle^{b}} f_{1}\left(\mu_{1}\right) f_{2}\left(\mu-\mu_{1}\right) f_{3}(\mu) d \mu d \mu_{1}\right| \lesssim \prod_{i=1}^{3}\left\|f_{i}\right\|_{L^{2}}
$$

where $k_{00}\left(\mu_{1}, \mu\right)=\langle\xi\rangle^{s}\left\langle\xi_{1}\right\rangle^{-s}\left\langle\xi-\xi_{1}\right\rangle^{-s}|\xi|\left|\xi_{1}\right|^{\frac{1}{2}}\left|\xi-\xi_{1}\right|^{-\frac{\alpha}{4}}$. On $A_{00}$ we have that $\left|\xi_{1}\right| \leq\left|\xi-\xi_{1}\right| \leq 1$ and therefore also $|\xi| \leq 2\left|\xi-\xi_{1}\right| \leq 2$, so that $k_{00}\left(\mu_{1}, \mu\right) \lesssim$ $|\xi|\left|\xi_{1}\right|^{\frac{1}{2}}\left|\xi-\xi_{1}\right|^{-\frac{\alpha}{4}} \lesssim\left|\xi-\xi_{1}\right|^{\frac{3}{2}-\frac{\alpha}{4}} \lesssim 1$, where the last inequality follows from $\alpha \leq 6$. Therefore (4.8) follows from the refined bilinear Strichartz estimate (3.13). 
Lemma 4.7. We have that

$$
\left\|Q_{10}\left(u_{1}, u_{2}\right)\right\|_{X_{1}^{s-(\alpha+1) b_{2}, b^{\prime}+b_{2}}} \lesssim\left\|u_{1}\right\|_{X_{0}^{s, b}}\left\|u_{2}\right\|_{X_{0}^{s, b}}
$$

provided that $b>\frac{1}{2}, b^{\prime}>-\frac{1}{2}$ and

$$
\begin{gathered}
0 \leq b_{2} \leq-b^{\prime}, \\
b^{\prime} \leq \frac{1}{\alpha+1}\left(\min (0, s)-\frac{3}{2}+\frac{\alpha}{4}\right) .
\end{gathered}
$$

Proof. We have to prove that

$$
\left|\int_{A_{10}} k_{10}\left(\mu_{1}, \mu\right) \frac{\left|\xi_{1}\right|^{-\frac{1}{2}}\left|\xi-\xi_{1}\right|^{\frac{\alpha}{4}}}{\left\langle\lambda_{1}\right\rangle^{b}\left\langle\lambda_{2}\right\rangle^{b}} f_{1}\left(\mu_{1}\right) f_{2}\left(\mu-\mu_{1}\right) f_{3}(\mu) d \mu d \mu_{1}\right| \lesssim \prod_{i=1}^{3}\left\|f_{i}\right\|_{L^{2}},
$$

where $k_{10}\left(\mu_{1}, \mu\right)=\langle\lambda\rangle^{b^{\prime}+b_{2}}\langle\xi\rangle^{1+s-(\alpha+1) b_{2}}\left\langle\xi_{1}\right\rangle^{-s}\left\langle\xi-\xi_{1}\right\rangle^{-s}\left|\xi_{1}\right|^{\frac{1}{2}}\left|\xi-\xi_{1}\right|^{-\frac{\alpha}{4}}$. We show that $k_{10}$ is bounded in $A_{10}$; then the lemma follows from the refined bilinear Strichartz estimate (3.11). In region $A_{10}$ we have $1 \leq\left|\xi-\xi_{1}\right| \sim|\xi| \sim\langle\xi\rangle$ and $|\lambda|=$ $\left|\lambda_{\max }\right| \gtrsim\left|\xi_{1}\right||\xi|^{\alpha}$, so using (4.10) we get $k_{10}\left(\mu_{1}, \mu\right) \lesssim|\xi|^{1-\frac{\alpha}{4}+\alpha b^{\prime}-b_{2}}\left\langle\xi_{1}\right\rangle^{-s}\left|\xi_{1}\right|^{\frac{1}{2}+b^{\prime}+b_{2}}$. Because of (4.10) we have $\frac{1}{2}+b^{\prime}+b_{2} \geq 0$. So using $\left|\xi_{1}\right| \leq|\xi| \sim\langle\xi\rangle$ it follows that $k_{10}\left(\mu_{1}, \mu\right) \lesssim\langle\xi\rangle^{\frac{3}{2}-\frac{\alpha}{4}+(\alpha+1) b^{\prime}-\min (0, s)} \lesssim 1$, where the last inequality follows from (4.11).

Lemma 4.8. We have that

$$
\left\|Q_{12}\left(u_{1}, u_{2}\right)\right\|_{X_{1}^{s-(\alpha+1) b_{2}, b^{\prime}+b_{2}}} \lesssim\left\|u_{1}\right\|_{X_{0}^{s, b}}\left\|u_{2}\right\|_{X_{0}^{s, b}}
$$

provided that $b>\frac{1}{2}, b^{\prime}>-\frac{1}{2}$ and (4.10) and (4.11) hold.

Proof. We have to show that

$$
\left|\int_{A_{12}} k_{12}\left(\mu_{1}, \mu\right) \frac{\left|\xi_{1}\right|^{-\frac{1}{2}}|\xi|^{\frac{\alpha}{4}}}{\left\langle\lambda_{1}\right\rangle^{b}\langle\lambda\rangle^{b}} f_{1}\left(\mu_{1}\right) f_{2}\left(\mu-\mu_{1}\right) f_{3}(\mu) d \mu d \mu_{1}\right| \lesssim \prod_{i=1}^{3}\left\|f_{i}\right\|_{L^{2}}
$$

where $k_{12}\left(\mu_{1}, \mu\right)=\langle\lambda\rangle^{b^{\prime}+b_{2}+b}\left\langle\lambda_{2}\right\rangle^{-b}\langle\xi\rangle^{1+s-(\alpha+1) b_{2}}\left\langle\xi_{1}\right\rangle^{-s}\left\langle\xi-\xi_{1}\right\rangle^{-s}\left|\xi_{1}\right|^{\frac{1}{2}}|\xi|^{-\frac{\alpha}{4}}$. Now using $b^{\prime}+b_{2}+b \geq 0$ and $|\lambda| \leq\left|\lambda_{2}\right|$ in $A_{12}$ we get $\langle\lambda\rangle^{b^{\prime}+b_{2}+b}\left\langle\lambda_{2}\right\rangle^{-b} \leq\left\langle\lambda_{2}\right\rangle^{b^{\prime}+b_{2}}$. Now the boundedness of $k_{12}$ on $A_{12}$ follows exactly like the boundedness of $k_{10}$ in Lemma 4.7. Then (4.12) follows from the refined bilinear Strichartz estimate (3.14).

Lemma 4.9. We have that

$$
\left\|Q_{11}\left(u_{1}, u_{2}\right)\right\|_{X_{1}^{s, 0}} \lesssim\left\|u_{1}\right\|_{X_{0}^{s, b-b^{\prime}}}\left\|u_{2}\right\|_{X_{0}^{s, b}}
$$

and

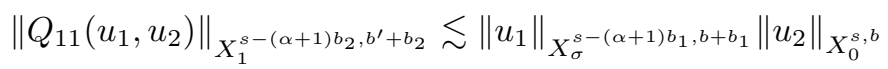

provided that $b>\frac{1}{2}, b^{\prime}>-\frac{1}{2}$, (4.10) and (4.11) hold and

$$
\begin{gathered}
0 \leq b_{2} \leq b_{1}, \\
\sigma \geq b_{1}-b^{\prime} \geq \frac{3}{2 \alpha}-\frac{1}{4} .
\end{gathered}
$$


Proof. We first show (4.13). We have to prove that

$$
\left|\int_{A_{11}} \tilde{k}_{11}\left(\mu_{1}, \mu\right) \frac{\left|\xi_{1}\right|^{-\frac{1}{2}}\left|\xi-\xi_{1}\right|^{\frac{\alpha}{4}}}{\left\langle\lambda_{1}\right\rangle^{b}\left\langle\lambda_{2}\right\rangle^{b}} f_{1}\left(\mu_{1}\right) f_{2}\left(\mu-\mu_{1}\right) f_{3}(\mu) d \mu d \mu_{1}\right| \lesssim \prod_{i=1}^{3}\left\|f_{i}\right\|_{L^{2}}
$$

where $\tilde{k}_{11}\left(\mu_{1}, \mu\right)=\left\langle\lambda_{1}\right\rangle^{b^{\prime}}\langle\xi\rangle^{1+s}\left\langle\xi_{1}\right\rangle^{-s}\left\langle\xi-\xi_{1}\right\rangle^{-s}\left|\xi_{1}\right|^{\frac{1}{2}}\left|\xi-\xi_{1}\right|^{-\frac{\alpha}{4}}$. We will show that $\tilde{k}_{11}$ is bounded; then (4.13) follows from the refined bilinear Strichartz estimate (3.11). In the region $A_{11}$ we have $1 \leq\left|\xi-\xi_{1}\right| \sim|\xi| \sim\langle\xi\rangle$ and $\left|\lambda_{1}\right|=\left|\lambda_{\max }\right| \gtrsim$ $\left|\xi_{1}\right||\xi|^{\alpha}$. Because of $b^{\prime} \leq 0$ we have $\tilde{k}_{11}\left(\mu_{1}, \mu\right) \lesssim|\xi|^{1-\frac{\alpha}{4}+\alpha b^{\prime}}\left\langle\xi_{1}\right\rangle^{-s}\left|\xi_{1}\right|^{\frac{1}{2}+b^{\prime}} \lesssim 1$, where the last inequality follows exactly as in the proof of Lemma 4.7 .

Now we show (4.14). We have to prove that

$$
\left|\int_{A_{11}} k_{11}\left(\mu_{1}, \mu\right) \frac{|\xi|^{-\frac{1}{4}+\frac{\alpha}{8}}\left|\xi-\xi_{1}\right|^{-\frac{1}{4}+\frac{\alpha}{8}}}{\langle\lambda\rangle^{b}\left\langle\lambda_{2}\right\rangle^{b}} f_{1}\left(\mu_{1}\right) f_{2}\left(\mu-\mu_{1}\right) f_{3}(\mu) d \mu d \mu_{1}\right| \lesssim \prod_{i=1}^{3}\left\|f_{i}\right\|_{L^{2}},
$$

where

$$
k_{11}\left(\mu_{1}, \mu\right)=\frac{\langle\xi\rangle^{1+s-(\alpha+1) b_{2}}\left\langle\xi_{1}\right\rangle^{-s+(\alpha+1) b_{1}-\sigma}\left\langle\xi-\xi_{1}\right\rangle^{-s}|\xi|^{\frac{1}{4}-\frac{\alpha}{8}}\left|\xi_{1}\right|^{\sigma}\left|\xi-\xi_{1}\right|^{\frac{1}{4}-\frac{\alpha}{8}}}{\langle\lambda\rangle^{-b^{\prime}-b_{2}-b}\left\langle\lambda_{1}\right\rangle^{b+b_{1}}} .
$$

Now (4.14) follows from the bilinear Strichartz estimate (3.6) if we show that $k_{11}$ is bounded on $A_{11}$. Because of $|\lambda| \leq\left|\lambda_{1}\right|$ and $-b^{\prime}-b_{2}-b \leq 0$, we have

$$
\langle\lambda\rangle^{-b^{\prime}-b_{2}-b}\left\langle\lambda_{1}\right\rangle^{b+b_{1}} \geq\left\langle\lambda_{1}\right\rangle^{-b^{\prime}+b_{1}-b_{2}} .
$$

In $A_{11}$ we have $1 \leq\left|\xi-\xi_{1}\right| \sim|\xi| \sim\langle\xi\rangle$ and $\left|\lambda_{1}\right| \gtrsim\left|\xi_{1}\right||\xi|^{\alpha}$, so using $-b^{\prime}+b_{1}-b_{2} \geq 0$ we get

$$
k_{11}\left(\mu_{1}, \mu\right) \lesssim|\xi|^{\frac{3}{2}-\frac{\alpha}{4}+\alpha\left(b^{\prime}-b_{1}\right)-b_{2}}\left\langle\xi_{1}\right\rangle^{-s+(\alpha+1) b_{1}-\sigma}\left|\xi_{1}\right|^{\sigma+b^{\prime}-b_{1}+b_{2}} .
$$

Because of $\left|\xi_{1}\right| \leq|\xi|$ in $A_{11}$, we have $|\xi|^{-b_{2}}\left|\xi_{1}\right|^{b_{2}} \leq 1$. By (4.16) we have $\sigma+b^{\prime}-b_{1} \geq$ 0 . If $\left|\xi_{1}\right| \leq 1$ we have $k_{11}\left(\mu_{1}, \mu\right) \lesssim|\xi|^{\frac{3}{2}-\frac{\alpha}{4}+\alpha\left(b^{\prime}-b_{1}\right)} \lesssim 1$, where the last inequality follows by (4.16). If $\left|\xi_{1}\right| \geq 1$ we have $\left\langle\xi_{1}\right\rangle \sim\left|\xi_{1}\right|$ and therefore

$$
k_{11}\left(\mu_{1}, \mu\right) \lesssim|\xi|^{\frac{3}{2}-\frac{\alpha}{4}+\alpha\left(b^{\prime}-b_{1}\right)}\left\langle\xi_{1}\right\rangle^{-s+b^{\prime}+\alpha b_{1}} .
$$

Now if $-s+b^{\prime}+\alpha b_{1} \leq 0$ this term is bounded because of (4.16) as above. If $-s+b^{\prime}+\alpha b_{1}>0$ this term is bounded by $c|\xi|^{\frac{3}{2}-\frac{\alpha}{4}-s+(\alpha+1) b^{\prime}}$ which is bounded because of (4.11).

Lemma 4.10. We have that

$$
\left\|Q_{20}\left(u_{1}, u_{2}\right)\right\|_{X_{\sigma}^{s-(\alpha+1) b_{2}, b^{\prime}+b_{2}}} \lesssim\left\|u_{1}\right\|_{X_{0}^{s, b}}\left\|u_{2}\right\|_{X_{0}^{s, b}}
$$

provided that $b>\frac{1}{2}, b^{\prime}>-\frac{1}{2}, b_{1} \leq-b^{\prime}$ and (4.11) hold and

$$
\begin{gathered}
0 \leq b_{2} \leq b_{1} \leq-b^{\prime}+\frac{1}{\alpha}\left(2 s-\frac{1}{2}+\frac{\alpha}{4}\right), \\
\sigma \leq 1+b^{\prime}+b_{1} .
\end{gathered}
$$

Proof. We have to show that

$$
\left|\int_{A_{20}} k_{20}\left(\mu_{1}, \mu\right) \frac{\left|\xi_{1}\right|^{-\frac{1}{4}+\frac{\alpha}{8}}\left|\xi-\xi_{1}\right|^{-\frac{1}{4}+\frac{\alpha}{8}}}{\left\langle\lambda_{1}\right\rangle^{b}\left\langle\lambda_{2}\right\rangle^{b}} f_{1}\left(\mu_{1}\right) f_{2}\left(\mu-\mu_{1}\right) f_{3}(\mu) d \mu d \mu_{1}\right| \lesssim \prod_{i=1}^{3}\left\|f_{i}\right\|_{L^{2}},
$$


where $k_{20}\left(\mu_{1}, \mu\right)=\langle\lambda\rangle^{b^{\prime}+b_{2}}\langle\xi\rangle^{s-(\alpha+1) b_{2}+\sigma}\left\langle\xi_{1}\right\rangle^{-s}\left\langle\xi-\xi_{1}\right\rangle^{-s}|\xi|^{1-\sigma}\left|\xi_{1}\right|^{\frac{1}{4}-\frac{\alpha}{8}}\left|\xi-\xi_{1}\right|^{\frac{1}{4}-\frac{\alpha}{8}}$. Now if we show that $k_{20}$ is bounded on $A_{20}$, the lemma follows from (3.4). In $A_{20}$ we have $1 \leq\left|\xi-\xi_{1}\right| \sim\left|\xi_{1}\right| \sim\left\langle\xi_{1}\right\rangle$ and $|\lambda|=\left|\lambda_{\max }\right|$, so

$$
k_{20}\left(\mu_{1}, \mu\right) \lesssim h\left(\mu_{1}, \mu\right):=\left\langle\lambda_{\max }\right\rangle^{b^{\prime}+b_{2}}\langle\xi\rangle^{s-(\alpha+1) b_{2}+\sigma}|\xi|^{1-\sigma}\left|\xi_{1}\right|^{-2 s+\frac{1}{2}-\frac{\alpha}{4}} .
$$

We will now show that $h$ is bounded in $\Xi_{2}$. Let us first consider the case that $|\xi| \geq 1$. Then because of $\left|\lambda_{\max }\right| \geq|\xi|\left|\xi_{1}\right|^{\alpha}$ and $\langle\xi\rangle \sim|\xi|$ we have

$$
h\left(\mu_{1}, \mu\right) \lesssim|\xi|^{1+s-\alpha b_{2}+b^{\prime}}\left|\xi_{1}\right|^{-2 s+\frac{1}{2}-\frac{\alpha}{4}+\alpha\left(b^{\prime}+b_{2}\right)} \lesssim|\xi|^{-s+\frac{3}{2}-\frac{\alpha}{4}+(\alpha+1) b^{\prime}},
$$

where the last inequality follows from (4.18) and $\left|\xi_{1}\right| \gtrsim|\xi|$. Now $h$ is bounded because of (4.11) and $|\xi| \geq 1$. So let us now consider the case $|\xi| \leq 1$. Because of (4.18) it follows that $\left\langle\lambda_{\max }\right\rangle^{b^{\prime}+b_{2}} \leq\left\langle\lambda_{\max }\right\rangle^{b^{\prime}+b_{1}}$ and therefore

$$
h\left(\mu_{1}, \mu\right) \lesssim|\xi|^{1-\sigma+b^{\prime}+b_{1}}\left|\xi_{1}\right|^{-2 s+\frac{1}{2}-\frac{\alpha}{4}+\alpha\left(b^{\prime}+b_{1}\right)} .
$$

Because of (4.19) and $|\xi| \leq 1$ we have $|\xi|^{1-\sigma+b^{\prime}+b_{1}} \lesssim 1$. Because of (4.18) we have $\left|\xi_{1}\right|^{-2 s+\frac{1}{2}-\frac{\alpha}{4}+\alpha\left(b^{\prime}+b_{1}\right)} \lesssim 1$. So $h$ is also bounded in this case, which proves the lemma.

Lemma 4.11. We have that

$$
\left\|Q_{21}\left(u_{1}, u_{2}\right)\right\|_{X_{\sigma}^{s-(\alpha+1) b_{2}, b^{\prime}+b_{2}}} \lesssim\left\|u_{1}\right\|_{X_{0}^{s, b}}\left\|u_{2}\right\|_{X_{0}^{s, b}}
$$

provided that $b>\frac{1}{2}, b^{\prime}>-\frac{1}{2}, b_{1} \leq-b^{\prime}$, (4.11), (4.18) and (4.19) hold.

Proof. We have to prove that

$$
\left|\int_{A_{21}} k_{21}\left(\mu_{1}, \mu\right) \frac{|\xi|^{-\frac{1}{2}}\left|\xi-\xi_{1}\right|^{\frac{\alpha}{4}}}{\langle\lambda\rangle^{b}\left\langle\lambda_{2}\right\rangle^{b}} f_{1}\left(\mu_{1}\right) f_{2}\left(\mu-\mu_{1}\right) f_{3}(\mu) d \mu d \mu_{1}\right| \lesssim \prod_{i=1}^{3}\left\|f_{i}\right\|_{L^{2}},
$$

where $k_{21}\left(\mu_{1}, \mu\right)=\left\langle\lambda_{1}\right\rangle^{-b}\langle\lambda\rangle^{b+b^{\prime}+b_{2}}\langle\xi\rangle^{s-(\alpha+1) b_{2}+\sigma}\left\langle\xi_{1}\right\rangle^{-s}\left\langle\xi-\xi_{1}\right\rangle^{-s}|\xi|^{\frac{3}{2}-\sigma}\left|\xi-\xi_{1}\right|^{-\frac{\alpha}{4}}$. Now if we show that $k_{21}$ is bounded in $A_{21}$, the lemma follows from the refined bilinear Strichartz estimate (3.15). In $A_{21}$ we have $|\lambda| \leq\left|\lambda_{\max }\right|=\left|\lambda_{1}\right|$ so that $\left\langle\lambda_{1}\right\rangle^{-b}\langle\lambda\rangle^{b+b^{\prime}+b_{2}} \leq\left\langle\lambda_{\max }\right\rangle^{b^{\prime}+b_{2}}$. Using that in $A_{21}$ we also have $1 \leq\left|\xi-\xi_{1}\right| \sim$ $\left|\xi_{1}\right| \sim\left\langle\xi_{1}\right\rangle$ we get

$$
\begin{aligned}
k_{21}\left(\mu_{1}, \mu\right) & \lesssim\left\langle\lambda_{\max }\right\rangle^{b^{\prime}+b_{2}}\langle\xi\rangle^{s-(\alpha+1) b_{2}+\sigma}|\xi|^{\frac{3}{2}-\sigma}\left|\xi_{1}\right|^{-2 s-\frac{\alpha}{4}} \\
& \lesssim\left\langle\lambda_{\max }\right\rangle^{b^{\prime}+b_{2}}\langle\xi\rangle^{s-(\alpha+1) b_{2}+\sigma}|\xi|^{1-\sigma}\left|\xi_{1}\right|^{-2 s+\frac{1}{2}-\frac{\alpha}{4}}
\end{aligned}
$$

where the last inequality follows because of $|\xi| \lesssim\left|\xi_{1}\right|$ in $A_{21}$. But this last expression is $h$ defined in (4.20) which was shown to be bounded in all of $\Xi_{2}$ in Lemma 4.10. This proves the lemma.

Lemma 4.12. We have that

$$
\left\|Q_{22}\left(u_{1}, u_{2}\right)\right\|_{X_{\sigma}^{s-(\alpha+1) b_{2}, b^{\prime}+b_{2}}} \lesssim\left\|u_{1}\right\|_{X_{0}^{s, b}}\left\|u_{2}\right\|_{X_{0}^{s, b}}
$$

provided that $b>\frac{1}{2}, b^{\prime}>-\frac{1}{2}, b_{1} \leq-b^{\prime}$, (4.11), (4.18) and (4.19) hold.

Proof. By definition (2.2) and duality we have to show that

$$
\left|\int_{A_{22}} k_{22}\left(\mu_{1}, \mu\right) \frac{|\xi|^{-\frac{1}{2}}\left|\xi_{1}\right|^{\frac{\alpha}{4}}}{\langle\lambda\rangle^{b}\left\langle\lambda_{1}\right\rangle^{b}} f_{1}\left(\mu_{1}\right) f_{2}\left(\mu-\mu_{1}\right) f_{3}(\mu) d \mu d \mu_{1}\right| \lesssim \prod_{i=1}^{3}\left\|f_{i}\right\|_{L^{2}},
$$

where $k_{22}\left(\mu_{1}, \mu\right)=\left\langle\lambda_{2}\right\rangle^{-b}\langle\lambda\rangle^{b+b^{\prime}+b_{2}}\langle\xi\rangle^{s-(\alpha+1) b_{2}+\sigma}\left\langle\xi_{1}\right\rangle^{-s}\left\langle\xi-\xi_{1}\right\rangle^{-s}|\xi|^{\frac{3}{2}-\sigma}\left|\xi_{1}\right|^{-\frac{\alpha}{4}}$. Now if we show that $k_{22}$ is bounded on $A_{22}$, the lemma follows from (3.16). But 
the boundedness of $k_{22}$ follows in exactly the same way as the boundedness of $k_{21}$ in Lemma 4.11 .

We are now in a position to prove Theorem 4.1.

Proof of Theorem 4.1. With our definitions of $b, b^{\prime}, b_{1}$ and $\sigma$ we have that $b>\frac{1}{2}$, $b-b^{\prime}<1, b_{1} \leq-b^{\prime}$, (4.11), (4.16) and (4.19) hold. Also (4.10), (4.15) and (4.18) hold for $b_{2}=0$ as well as for $b_{2}=b_{1}$. We noticed before that because of the symmetry of (4.1) in $u_{1}$ and $u_{2}$, it suffices to show (4.1) for $\partial_{x} P_{1}\left(u_{1}, u_{2}\right)$ instead of $\partial_{x}\left(u_{1} u_{2}\right)$ where $P_{1}$ is the operator defined in (3.7). We now decompose $\partial_{x} P_{1}\left(u_{1}, u_{2}\right)$ further as in (4.5). Therefore we have to show for every $Q_{i j}$ that

$$
\left\|Q_{i j}\left(u_{1}, u_{2}\right)\right\|_{\tilde{X}} \leq C\left\|u_{1}\right\|_{X}\left\|u_{2}\right\|_{X} .
$$

Let us notice that by the definition of the space $\tilde{X}$ we have that $\|u\|_{\tilde{X}} \leq\|u\|_{X_{0}^{s, 0}}$ and $\|u\|_{\tilde{X}} \leq\|u\|_{X_{\sigma}^{s, b^{\prime}}}+\|u\|_{X_{\sigma}^{s-(\alpha+1) b_{1}, b^{\prime}+b_{1}}}$, so that it suffices to control $Q_{i j}$ in one of these norms. By (4.4) we see that (4.23) follows from

$$
\left\|Q_{i j}\left(u_{1}, u_{2}\right)\right\|_{\tilde{X}} \leq C\left\|u_{1}\right\|_{X_{0}^{s, b}}\left\|u_{2}\right\|_{X_{0}^{s, b}}
$$

which actually holds for all of the $Q_{i j}$ except $Q_{11}$. Let us prove this first. From Lemma 4.6 it follows that

$$
\begin{aligned}
\left\|Q_{00}\left(u_{1}, u_{2}\right)\right\|_{\tilde{X}} & \leq\left\|Q_{00}\left(u_{1}, u_{2}\right)\right\|_{X_{0}^{s, 0}} \\
& \lesssim\left\|u_{1}\right\|_{X_{0}^{s, b}}\left\|u_{2}\right\|_{X_{0}^{s, b}} \lesssim\left\|u_{1}\right\|_{X}\left\|u_{2}\right\|_{X} .
\end{aligned}
$$

In the same way, from Lemmas 4.7 4.8, 4.10, 4.11 and 4.12 it follows that

$$
\begin{aligned}
\left\|Q_{i j}\left(u_{1}, u_{2}\right)\right\|_{\tilde{X}} & \leq\left\|Q_{i j}\left(u_{1}, u_{2}\right)\right\|_{X_{\sigma}^{s, b^{\prime}}}+\left\|Q_{i j}\left(u_{1}, u_{2}\right)\right\|_{X_{\sigma}^{s-(\alpha+1) b_{1}, b^{\prime}+b_{1}}} \\
& \lesssim\left\|u_{1}\right\|_{X_{0}^{s, b}}\left\|u_{2}\right\|_{X_{0}^{s, b}} \lesssim\left\|u_{1}\right\|_{X}\left\|u_{2}\right\|_{X}
\end{aligned}
$$

for all of the remaining $Q_{i j}$ except $Q_{11}$. So it remains to consider $Q_{11}$. Now let us decompose $u_{1} \in X$ as $u_{1}=v_{1}+w_{1}$ with $v_{1} \in X_{1}$ and $w_{1} \in X_{2}$. For $v_{1}$ we have, because of (4.13) of Lemma 4.9.

$$
\begin{aligned}
\left\|Q_{11}\left(v_{1}, u_{2}\right)\right\|_{\tilde{X}} & \leq\left\|Q_{11}\left(u_{1}, u_{2}\right)\right\|_{X_{0}^{s, 0}} \\
& \lesssim\left\|v_{1}\right\|_{X_{0}^{s, b-b^{\prime}}}\left\|u_{2}\right\|_{X_{0}^{s, b}} \lesssim\left\|v_{1}\right\|_{X_{1}}\left\|u_{2}\right\|_{X} .
\end{aligned}
$$

For $w_{1}$ we have, because of (4.14) of Lemma 4.9.

$$
\begin{aligned}
\left\|Q_{11}\left(w_{1}, u_{2}\right)\right\|_{\tilde{X}} & \leq\left\|Q_{11}\left(w_{1}, u_{2}\right)\right\|_{X_{\sigma}^{s, b^{\prime}}}+\left\|Q_{11}\left(w_{1}, u_{2}\right)\right\|_{X_{\sigma}^{s-(\alpha+1) b_{1}, b^{\prime}+b_{1}}} \\
& \lesssim\left\|w_{1}\right\|_{X_{\sigma}^{s-(\alpha+1) b_{1}, b+b_{1}}}\left\|u_{2}\right\|_{X_{0}^{s, b}} \lesssim\left\|w_{1}\right\|_{X_{2}}\left\|u_{2}\right\|_{X} .
\end{aligned}
$$

So putting these two estimates together we have

$$
\left\|Q_{11}\left(u_{1}, u_{2}\right)\right\|_{\tilde{X}} \lesssim\left(\left\|v_{1}\right\|_{X_{1}}+\left\|w_{1}\right\|_{X_{2}}\right)\left\|u_{2}\right\|_{X} .
$$

Now taking the infimum over every decomposition of $u_{1}$ of the form $u_{1}=v_{1}+w_{1}$ with $v_{1} \in X_{1}$ and $w_{1} \in X_{2}$ we finally get (4.23) for $Q_{11}$, which finishes the proof.

For the proof of Theorem 1.6 we need the following refined version of (4.1).

Corollary 4.13. Let $\frac{4}{3}<\alpha \leq 6$ and $s>\max \left(1-\frac{3}{4} \alpha, \frac{1}{4}-\frac{3}{8} \alpha\right)$. Let $X$ and $\tilde{X}$ be defined as in Theorem 4.1. We then have for every $\rho>0$

$$
\left\|J_{x}^{\rho} \partial_{x}\left(u_{1} u_{2}\right)\right\|_{\tilde{X}} \lesssim\left(\left\|J_{x}^{\rho} u_{1}\right\|_{X}\left\|u_{2}\right\|_{X}+\left\|u_{1}\right\|_{X}\left\|J_{x}^{\rho} u_{2}\right\|_{X}\right) .
$$


Proof. Writing $\partial_{x}\left(u_{1} u_{2}\right)=\partial_{x} P_{1}\left(u_{1}, u_{2}\right)+\partial_{x} P_{1}\left(u_{2}, u_{1}\right)$, where $P_{1}$ is the operator defined in (3.7), it suffices to show that

$$
\left\|J_{x}^{\rho} \partial_{x} P_{1}\left(u_{1}, u_{2}\right)\right\|_{\tilde{X}} \lesssim\left\|u_{1}\right\|_{X}\left\|J_{x}^{\rho} u_{2}\right\|_{X} .
$$

But this follows exactly as (4.1), as the operators $J_{x}^{\rho}$ in (4.25) only give an additional bounded term $\left(\frac{\langle\xi\rangle}{\left\langle\xi-\xi_{1}\right\rangle}\right)^{\rho}$ in the dual formulations of the estimates proved in Lemmas 4.64.12,

\section{Proof of Theorems 1.2 and 1.6}

As the methods of proof used here are all well known, we only give

Sketch of the proof of Theorem 1.2. As explained at the beginning of Section 4 it suffices to consider the case $s_{2}=0$. Let $s=s_{1}$. For $T \leq 1$ and $u_{1}, u_{2} \in \tilde{\mathcal{S}}$ we define the bilinear operator $\Gamma_{T}$ by (1.8). Let $X$ and $\tilde{X}$ be defined as in Theorem 4.1 and set $\delta:=1-\left(b-b^{\prime}\right)>0$. Then by (4.3) and Theorem 4.1 we have

$$
\left\|\Gamma_{T}\left(u_{1}, u_{2}\right)\right\|_{X} \lesssim T^{\delta}\left\|\partial_{x}\left(u_{1} u_{2}\right)\right\|_{\tilde{X}} \lesssim T^{\delta}\left\|u_{1}\right\|_{X}\left\|u_{2}\right\|_{X} .
$$

Therefore we can extend $\Gamma_{T}$ to a continuous, bilinear operator $\Gamma_{T}: X \times X \rightarrow X$. As $\Gamma_{T}\left(u_{1}, u_{2}\right)_{\mid[-T, T]}$ only depends on $u_{i \mid[-T, T]}(i=1,2), \Gamma_{T}$ also defines a continuous, bilinear operator $\Gamma_{T}: X_{T} \times X_{T} \rightarrow X_{T}$. Furthermore by (4.2) we have

$$
\left\|\psi U_{\alpha}(\cdot) u_{0}\right\|_{X_{T}} \leq\left\|\psi U_{\alpha}(\cdot) u_{0}\right\|_{X} \lesssim\left\|u_{0}\right\|_{H^{s, 0}\left(\mathbb{R}^{2}\right)}
$$

for $u_{0} \in H^{s, 0}\left(\mathbb{R}^{2}\right)$. So if we define

$$
\Phi_{T}\left(u, u_{0}\right):=\psi U_{\alpha}(\cdot) u_{0}-\Gamma_{T}(u, u), \quad u \in X_{T}, \quad u_{0} \in H^{s, 0}\left(\mathbb{R}^{2}\right),
$$

we have for $u_{0} \in B_{R}:=\left\{u_{0} \in H^{s, 0}\left(\mathbb{R}^{2}\right) \mid\left\|u_{0}\right\|_{H^{s, 0}}<R\right\}$ and $u, v \in \bar{A}_{r}:=\{u \in$ $\left.X_{T} \mid\|u\|_{X_{T}} \leq r\right\}$ that

$$
\left\|\Phi_{T}\left(u, u_{0}\right)\right\|_{X_{T}} \leq C\left(\left\|u_{0}\right\|_{H^{s, 0}\left(\mathbb{R}^{2}\right)}+T^{\delta}\|u\|_{X_{T}}^{2}\right) \leq C R+C T^{\delta} r^{2}
$$

with some constant $C$ which does not depend on $R, r$ and $T$, and

$$
\begin{array}{r}
\left\|\Phi_{T}\left(u, u_{0}\right)-\Phi_{T}\left(v, u_{0}\right)\right\|_{X_{T}}=\left\|\Gamma_{T}(u-v, u+v)\right\|_{X_{T}} \\
\leq C T^{\delta}\left(\|u\|_{X_{T}}+\|v\|_{X_{T}}\right)\|u-v\|_{X_{T}} \leq 2 C T^{\delta} r\|u-v\|_{X_{T}} .
\end{array}
$$

So given $R>0$ we choose $r=2 C R$ and $T=\min \left\{1,\left(8 C^{2} R\right)^{-\frac{1}{\delta}}\right\}$. Then, for fixed $u_{0} \in B_{R}$, by (5.2) $\Phi_{T}\left(\cdot, u_{0}\right)$ maps $\bar{A}_{r}$ into $\bar{A}_{r}$ and by (5.3) $\Phi_{T}\left(u_{0}, \cdot\right)$ is a contraction. By the Banach fixed point theorem there is exactly one fixed point of $\Phi_{T}$ in $\bar{A}_{r}$. Now by a well known argument the uniqueness of the solution $u$ also follows in $X_{T}$. Furthermore it is easy to see that the mapping

$$
\Lambda_{T}: X_{T} \times B_{R} \rightarrow X_{T}, \quad \Lambda_{T}\left(u, u_{0}\right):=\Phi_{T}\left(u, u_{0}\right)-u
$$

is analytic. Therefore a standard use of the implicit function theorem yields the analyticity of the flow map $F_{R}: u_{0} \mapsto u$.

Sketch of the proof of Theorem 1.6. Let $u_{0} \in H^{s, 0}\left(\mathbb{R}^{2} ; \mathbb{R}\right)$ be real valued and let $X$ be defined as in Theorem 4.1, Let $T_{0}$ be the supremum of all $T \in(0,1]$ such that there exists a unique $u \in X_{T}$ with $\Phi_{T}\left(u, u_{0}\right)=u$. We will prove that $T_{0}=1$. By Theorem 1.2 we see that $T_{0}>0$. Let $T \in\left(0, T_{0}\right)$. Let $X^{0}$ be defined as $X$ in Theorem 4.1, but with $s=0$. We obviously have $\|u\|_{X}=\left\|J^{s} u\right\|_{X^{0}}$. By (4.2), (4.3) and Corollary 4.13 we have

$$
\left\|J^{s} u\right\|_{X_{T}^{0}} \leq C R+2 C T^{\delta}\|u\|_{X_{T}^{0}}\left\|J^{s} u\right\|_{X_{T}^{0}} .
$$


Now if $T_{0} \leq \min \left(1,\left(8 C^{2}\left\|u_{0}\right\|_{L^{2}}\right)^{-\frac{1}{\delta}}\right)$ we have by (5.2) that $\|u\|_{X_{T}^{0}} \leq 2 C\left\|u_{0}\right\|_{L^{2}}$ and therefore

$$
\left\|J^{s} u\right\|_{X_{T}^{0}} \leq C R+\frac{1}{2}\left\|J^{s} u\right\|_{X_{T}^{0}} .
$$

It follows that $\sup _{|t|<T}\|u(t)\|_{H^{s, 0}} \leq \tilde{C}\left\|J^{s} u\right\|_{X_{T}^{0}} \leq 2 \tilde{C} C R$. As this upper bound does not depend on $\bar{T}$ and applying Theorem 1.2 with $u(T)$ and $u(-T)$ as initial values, we see that we can extend the solution beyond the interval $\left[-T_{0}, T_{0}\right]$. This contradicts the choice of $T_{0}$. Therefore we have $T_{0} \geq \min \left(1,\left(8 C^{2}\left\|u_{0}\right\|_{L^{2}}\right)^{-\frac{1}{\delta}}\right)$. This implies that the length of the maximal interval of existence does only depend on $\left\|u_{0}\right\|_{L^{2}}$. But the $L^{2}$-norm of real valued solutions $u$ of (1.7) is conserved, i.e. $\|u( \pm T)\|_{L^{2}}=\left\|u_{0}\right\|_{L^{2}}$, so if we had $T_{0}<1$, we could extend the solution beyond the interval $\left[-T_{0}, T_{0}\right]$ which contradicts the choice of $T_{0}$.

\section{REFERENCES}

1. J. Bourgain, Fourier transform restriction phenomena for certain lattice subsets and applications to nonlinear evolution equations. I. Schrödinger equations, Geom. Funct. Anal. 3 (1993), no. 2, 107-156. MR.1209299 (95d:35160a)

2. _ Fourier transform restriction phenomena for certain lattice subsets and applications to nonlinear evolution equations. II. The KdV-equation, Geom. Funct. Anal. 3 (1993), no. 3, 209-262. MR1215780 (95d:35160b)

3. 3 (1993), no. 4, 315-341. MR1223434 (94d:35142)

4. Jean Ginibre, Le problème de Cauchy pour des EDP semi-linéaires périodiques en variables d'espace (d'après Bourgain), Astérisque (1996), no. 237, Exp. No. 796, 4, 163-187, Séminaire Bourbaki, Vol. 1994/95. MR.1423623 (98e:35154)

5. R. J. Iório, Jr. and W. V. L. Nunes, On equations of KP-type, Proc. Roy. Soc. Edinburgh Sect. A 128 (1998), no. 4, 725-743. MR.1635416 (99j:35189)

6. P. Isaza and J. Mejía, Local and global Cauchy problems for the Kadomtsev-Petviashvili (KPII) equation in Sobolev spaces of negative indices, Comm. Partial Differential Equations 26 (2001), no. 5-6, 1027-1054. MR.1843294 (2002e:35205)

7. Pedro Isaza, Juan López, and Jorge Mejía, Cauchy problem for the fifth order KadomtsevPetviashvili (KPII) equation, Commun. Pure Appl. Anal. 5 (2006), no. 4, 887-905. MR.2246014

8. B.B. Kadomtsev and V.I. Petviashvili, On the stability of solitary waves in weakly dispersing media, Sov. Phys., Dokl. 15 (1970), 539-541 (English. Russian original).

9. C. E. Kenig, G. Ponce, and L. Vega, Oscillatory integrals and regularity of dispersive equations, Indiana Univ. Math. J. 40 (1991), no. 1, 33-69. MR.1101221 (92d:35081)

10. C. E. Kenig and S. N. Ziesler, Local well posedness for modified Kadomstev-Petviashvili equations, Differential Integral Equations 18 (2005), no. 10, 1111-1146. MR2162626 (2006g:35236)

11. L. Molinet, J. C. Saut, and N. Tzvetkov, Remarks on the mass constraint for KP type equations, preprint, arXiv:math.AP/0603303, 2006.

12. J. C. Saut, Remarks on the generalized Kadomtsev-Petviashvili equations, Indiana Univ. Math. J. 42 (1993), no. 3, 1011-1026. MR.1254130 (95j:35200)

13. J. C. Saut and N. Tzvetkov, The Cauchy problem for higher-order KP equations, J. Differential Equations 153 (1999), no. 1, 196-222. MR1682263(2000c:35212)

14. (2000), no. 4, 307-338. MR1753060 (2001d:35175)

15. H. Takaoka, Global well-posedness for the Kadomtsev-Petviashvili II equation, Discrete Contin. Dynam. Systems 6 (2000), no. 2, 483-499. MR1739371 (2000m:35163)

16. W Well-posedness for the Kadomtsev-Petviashvili II equation, Adv. Differential Equations 5 (2000), nos. 10-12, 1421-1443. MR.1785680 (2001i:35255)

17. H. Takaoka and N. Tzvetkov, On the local regularity of the Kadomtsev-Petviashvili-II equation, Internat. Math. Res. Notices (2001), no. 2, 77-114. MR1810481(2001j:35243) 
18. N. Tzvetkov, On the Cauchy problem for Kadomtsev-Petviashvili equation, Comm. Partial Differential Equations 24 (1999), no. 7-8, 1367-1397. MR.1697491 (2000i:35181)

19. _ Global low-regularity solutions for Kadomtsev-Petviashvili equation, Differential Integral Equations 13 (2000), nos. 10-12, 1289-1320. MR.1787069 (2001g:35227)

Mathematical Institute of the University of Bonn, Beringstrasse 1, D-53115 Bonn, Germany

E-mail address: hadac@math.uni-bonn.de 